\title{
The Evolution of Inhomogeneous Wave Statistics through a Variable Medium
}

\author{
P. B. SMIT \\ Delft University of Technology, Delft, Netherlands \\ T. T. JANSSEN \\ Theiss Research, El Granada, California
}

(Manuscript received and in final form 1 March 2013)

\begin{abstract}
The interaction of ocean waves with variable currents and topography in coastal areas can result in inhomogeneous statistics because of coherent interferences, which affect wave-driven circulation and transport processes. Stochastic wave models, invariably based on some form of the radiative transfer equation (or action balance), do not account for these effects. The present work develops and discusses a generalization of the radiative transfer equation that includes the effects of coherent interferences on wave statistics. Using multiple scales, the study approximates the transport equation for the (complete) second-order wave correlation matrix. The resulting model transports the coupled-mode spectrum (a form of the Wigner distribution) and accounts for the generation and propagation of coherent interferences in a variable medium. The authors validate the model through comparison with analytic solutions and laboratory observations, discuss the differences with the radiative transfer equation and the limitations of this approximation, and illustrate its ability to resolve coherent interference structures in wave fields such as those typically found in refractive focal zones and around obstacles.
\end{abstract}

\section{Introduction}

The dynamics and statistics of ocean waves are important, for example, for upper-ocean dynamics (e.g., Craik and Leibovich 1976; Smith 2006; Aiki and Greatbatch 2011), air-sea interaction (e.g., Janssen 2009), ocean circulation (e.g., McWilliams and Restrepo 1999), and wave-driven circulation and transport processes (e.g., Hoefel and Elgar 2003; Svendsen 2006). Modern stochastic wave models are routinely applied to a wide range of oceanic scales, both in open-ocean applications and the near shore, and either as stand-alone wave prediction models, or as part of coupled ocean-atmosphere models for global circulation and climate studies (e.g., The WAMDI Group 1988; Tolman 1991; Komen et al. 1994; Booij et al. 1999; Wise Group 2007). These so-called third-generation wave models are invariably based on some form of the radiative transfer equation (or action balance)

Corresponding author address: Pieter Smit, Environmental Fluid Mechanics, Faculty of Civil Engineering and Geosciences, Delft University of Technology, Stevinweg 1, 2628 CN Delft, Netherlands. E-mail: p.b.smit@tudelft.nl

$$
\partial_{t} E+\mathbf{c}_{\mathbf{x}} \cdot \nabla_{\mathbf{x}} E+\mathbf{c}_{\mathbf{k}} \cdot \nabla_{\mathbf{k}} E=S
$$

which describes the evolution of the variance (or action) density spectrum $E(\mathbf{k}, \mathbf{x}, t)$ through time $t$, geographical space $\mathbf{x}$, and wavenumber space $\mathbf{k}$, with the transport velocities $\mathbf{c}_{\mathbf{x}}$ and $\mathbf{c}_{\mathbf{k}}$, respectively, and augmented with (parameterized) source terms $S(\mathbf{k}, \mathbf{x}, t)$ to account for nonconservative and nonlinear processes.

Continuing development of these models is generally through improvements of the source term parameterizations on the right side of Eq. (1), but the left side, the radiative transfer equation (RTE), has not changed since the early development of these models (e.g., The WAMDI Group 1988; Komen et al. 1994; Wise Group 2007). The RTE transports wave variance density through a slowly varying medium such that wave energy (or action) is conserved, while assuming that the wave field is (and remains) quasi-homogeneous and near-Gaussian. In the open ocean, where medium variations are generally very weak, and wave statistics evolve principally through the action of wind, dissipation (whitecapping) and third-order nonlinear effects, the assumptions of homogeneity and Gaussianity are often easily met. However, on continental 
shelves and in coastal regions, where wave fields travel through shallower water, and medium variations are stronger (both currents and topography), the wave field can develop and maintain inhomogeneities that strongly affect the wave statistics (e.g., Janssen et al. 2008; Janssen and Herbers 2009). For instance, the refraction over coastal topography or currents (e.g., Berkhoff et al. 1982; Vincent and Briggs 1989; Magne et al. 2007; Janssen et al. 2008), or diffraction around obstacles such as breakwaters, reefs, or headlands (e.g., Penney and Price 1952), can result in relatively fast variations in wave statistics because of coherent wave interference patterns. The effects of such coherent structures on the wave statistics are not accounted for by the RTE (Vincent and Briggs 1989; O'Reilly and Guza 1991).

To account for the effects of coherent interferences on the wave statistics, more general transport models for second-order wave statistics were developed in other fields, such as optics and quantum mechanics (e.g., Wigner 1932; Bremmer 1972; Bastiaans 1979; Cohen 2010). For ocean waves such models were developed for special cases, including narrowband waves (e.g., Alber 1978) and forward-scattered waves through a weakly two-dimensional medium (e.g., Janssen et al. 2008). In the present work, we apply the ideas developed in optics (e.g., Bremmer 1972; Bastiaans 1979; Cohen 2010) to derive a more general transport model for ocean wave statistics in the presence of caustics and coherent interferences, which includes the RTE as a special case.

Thereto we derive a general transport equation for the second-order correlation matrix for linear waves in a slowly varying medium, and-using multiple scalesderive a consistent, quasi-coherent approximation that includes coherent interferences (section 2). In section 3, to illustrate the accuracy of our approximations and the differences with the RTE, we compare a numerical implementation of the model with an analytic solution for the evolution of coherent Gaussian wave packets and compare model simulations with observations of random wave propagation over a two-dimensional shoal (Vincent and Briggs 1989). We discuss (section 4) the spectral distribution function (the coupled-mode spectrum), the limits of the approximation, wave diffraction in the quasi-coherent approximation, and show that the quasi-coherent approximation includes earlier results as special cases (e.g., Alber 1978).

\section{Evolution of correlators}

To study the generation and propagation of coherent structures in random ocean wave fields propagating through a variable medium, we consider the statistics of the free-surface elevation $\eta(\mathbf{x}, t)$, represented as a zero-mean random wave variable and a function of the horizontal coordinates $\mathbf{x}=(x, y)$ and time $t$. We define a complex variable $\zeta$, such that $\eta=\operatorname{Re}(\zeta)$, its real and imaginary parts form a Hilbert transform pair (see, e.g., Mandel and Wolf 1995), and its Fourier transform $\hat{\zeta}$ is defined by the transform pair ${ }^{1}$

$$
\begin{aligned}
& \zeta(\mathbf{x}, t)=\int \hat{\zeta}(\mathbf{k}, t) \exp (i \mathbf{k} \cdot \mathbf{x}) d \mathbf{k} \quad \text { and } \\
& \hat{\zeta}(\mathbf{k}, t)=\frac{1}{(2 \pi)^{2}} \int \zeta(\mathbf{x}, t) \exp (-i \mathbf{k} \cdot \mathbf{x}) d \mathbf{x} .
\end{aligned}
$$

We assume that medium variations are relatively slow, varying $O(1)$ over distances $l_{0} / \varepsilon$, with $l_{0}$ being a characteristic wavelength and $\varepsilon \ll 1$, so that plane wave solutions are admitted and a dispersion relation of the form $\omega=\sigma(\mathbf{k}, \mathbf{x})$ exists locally. The free-surface elevation $\eta$ is considered a superposition of slowly varying wave packets $\zeta_{j}$, each characterized by its position $\mathbf{x}_{j}(t)$, wavenumber $\mathbf{k}_{j}(t)$, and angular frequency $\omega_{j}(t)$. In the present work we consider medium variations owing to variations in depth $h(\mathbf{x})$, so that in absence of currents [and to $O(\varepsilon)$ ] the dispersion relation is $\sigma(\mathbf{k}, \mathbf{x})=$ $\sqrt{g k \tanh (k h)}$, where $k=|\mathbf{k}|$ (e.g., Dingemans 1997; Mei et al. 2005).

Our starting point is the equation of motion for the transformed free-surface variable $\hat{\zeta}(\mathbf{k}, t)$, which we write as (e.g., Salmon 1998; Bremmer 1972)

$$
\partial_{t} \hat{\zeta}(\mathbf{k}, t)=-i \Omega\left(\mathbf{k}, i \nabla_{\mathbf{k}}\right) \hat{\zeta}(\mathbf{k}, t)
$$

Here we use operator correspondence between conjugate variables: $-i \omega_{j} \rightarrow \partial_{t}, \mathbf{x}_{j} \rightarrow i \nabla_{\mathbf{k}}$, and $\mathbf{k}_{j} \rightarrow \mathbf{k}$, to relate the local dispersion relation to an operator $\sigma\left(\mathbf{k}_{j}, \mathbf{x}_{j}\right) \rightarrow$ $\Omega\left(\mathbf{k}, i \nabla_{\mathbf{k}}\right)$, which is defined using the Weyl correspondence rule [see appendix A, and, e.g., Agarwal and Wolf (1970)]. It can be readily shown that the wave Eq. (3) describes progressive waves (appendix A), is exact in a homogeneous medium (appendix A), and is consistent with WKB theory for slowly varying waves (appendix B). From the wave Eq. (3), an evolution equation for the second-order moments $\left\langle\hat{\zeta}_{1} \hat{\zeta}_{2}^{*}\right\rangle$ is obtained in the usual way, ${ }^{2}$ and upon transforming the coordinates to

\footnotetext{
${ }^{1}$ Unless made explicit otherwise, integration limits on Fourier integrals are from $-\infty$ to $+\infty$, and the transforms are assumed to exist in the context of generalized functions (Strichartz 1993).

${ }^{2}$ Multiply Eq. (3) for $\hat{\zeta}_{1}=\hat{\zeta}\left(\mathbf{k}_{1}, t\right)$ by $\hat{\zeta}_{2}^{*}=\hat{\zeta}\left(\mathbf{k}_{2}, t\right)$, and the equation for $\hat{\zeta}_{2}^{*}$ by $\hat{\zeta}_{1}$, sum both relations and ensemble average the result.
} 
$\mathbf{k}=\left(\mathbf{k}_{1}+\mathbf{k}_{2}\right) / 2$ and $\mathbf{u}=\mathbf{k}_{1}-\mathbf{k}_{2}$, and Fourier transforming the result with respect to the difference wavenumber $\mathbf{u}$, the transport equation for the second-order statistics can be written as (see e.g., Bremmer 1972; Bastiaans 1997; Cohen 2010)

$$
\begin{aligned}
\partial_{t} \mathcal{E}= & -i\left[\Omega\left(\mathbf{k}-\frac{i}{2} \boldsymbol{\nabla}_{\mathbf{x}}, \mathbf{x}+\frac{i}{2} \boldsymbol{\nabla}_{\mathbf{k}}\right)\right. \\
& \left.-\Omega\left(\mathbf{k}+\frac{i}{2} \nabla_{\mathbf{x}}, \mathbf{x}-\frac{i}{2} \nabla_{\mathbf{k}}\right)\right] \mathcal{E},
\end{aligned}
$$

with

$$
\mathcal{E}(\mathbf{k}, \mathbf{x}, t)=\frac{1}{2} \int\left\langle\hat{\zeta}\left(\mathbf{k}+\frac{\mathbf{u}}{2}, t\right) \hat{\zeta} *\left(\mathbf{k}-\frac{\mathbf{u}}{2}, t\right)\right\rangle \exp (i \mathbf{u} \cdot \mathbf{x}) d \mathbf{u} .
$$

Here, the angle brackets \langle\rangle denote ensemble averaging. The distribution function $\mathcal{E}$ represents the complete second-order statistics and carries the same information as the two-point correlator $\left\langle\hat{\zeta}_{1} \hat{\zeta}_{2}^{*}\right\rangle$, but with the wavenumber separation exchanged for a space coordinate. In appearance it is similar to the widely used variance density spectrum, and likewise, the local wave variance $\mathcal{V}(\mathbf{x}, t)$ is found from the marginal distribution

$$
\mathcal{V}(\mathbf{x}, t)=\int \mathcal{E}(\mathbf{k}, \mathbf{x}, t) d \mathbf{k} .
$$

However, the distribution function $\mathcal{E}$ is generally not point-wise positive, and can thus not be interpreted as a variance density function (see, e.g., Janssen and Claasen 1985). Only in the limit where the wave field is quasi-homogeneous is the spectrum $\mathcal{E}$ positive everywhere and reduces to a variance density function (which is thus a special case). To distinguish $\mathcal{E}$, as defined here, from the widely used variance density spectrum, we refer to it as a coupled-mode (CM) spectrum.

The evolution Eq. (4) describes the evolution of the $\mathrm{CM}$ spectrum through a variable medium and is exact in the WKB sense in that it does not make any assumption regarding the statistical homogeneity or the scales of variation of the wave statistics. In other words, although the wave components themselves are slowly varying (in accordance with WKB), the statistics can undergo rapid variations (on the scale of individual wave lengths) through the development and propagation of cross correlations in the wave field, either through the interaction with medium variations, or radiated in from the boundaries.

\section{An approximate transport model for inhomogeneous} wave fields

The transport Eq. (4) governs the evolution of the CM spectrum within a slowly varying medium, but apart from certain special cases, the dynamical implications of the operators in Eq. (4) are not easily understood, and they cannot be readily numerically evaluated. To derive a consistent approximation to these operators, we introduce the following coordinate scaling. We define two (independent) spatial scales: a slow scale $\mathbf{X}_{m}$ associated with the medium variations, and a scale $\mathbf{X}$ that captures the spatial variations of the spectrum as a result of the wavenumber mismatches in the cross correlations, written as

$$
\mathbf{X}_{m}=\varepsilon x \quad \text { and } \quad \mathbf{X}=\mu x
$$

respectively. Here $\mu=\Delta u / k_{0}\left(\right.$ where $\left.k_{0}=2 \mu / l_{0}\right)$, with $\Delta u$ a representative difference wavenumber for the cross correlations and $k_{0}$ a characteristic wavenumber of the wave field. Because we consider cross correlations developed through the interaction with a slowly varying medium, we have $\mu \ll 1$ (and $\mu$ is generally of the same order as $\varepsilon$ ), so that $\mathbf{X}$ is effectively a slow scale [with $O(1)$ variations on length scales of $\left.l_{0} / \mu\right]$. To make the width of the spectrum explicit, we consider the wavenumber scale

$$
\mathbf{K}=\delta^{-1} \mathbf{k},
$$

where $\delta=\Delta k / k_{0}$, with $\Delta k$ representing a characteristic width of the spectrum. The $\delta$ can be thought of as an inverse correlation length scale so that for a highly coherent (narrow band) wave field $\delta \ll 1$ (and $\mathbf{K}$ is a fast scale), whereas for moderate- or wide-band wave fields $\delta \sim O(1)$. Lastly, we introduce the time scales

$$
T_{j}=\mu^{j} t ; \quad j=1,2, \ldots N \in \mathbb{Z} .
$$

Using these scales, the dependent variable $\mathcal{E}$ and the dispersion relation $\sigma$ become

$$
\mathcal{E}=\mathcal{E}\left(\mathbf{K}, \mathbf{X}, T_{1}, T_{2}, \ldots, T_{N}\right) \quad \text { and } \quad \sigma=\sigma\left(\mathbf{k}, \mathbf{X}_{m}\right),
$$

so that, to $O\left(\mu^{N}\right)$, the governing Eq. (4) can be expressed as

$$
\begin{aligned}
\mu^{j} \partial_{T_{j}} \mathcal{E}= & -i\left[\Omega\left(\mathbf{k}-\mu \frac{i}{2} \nabla_{\mathbf{X}}, \mathbf{X}_{m}+\delta \frac{i}{2} \nabla_{\mathbf{K}}\right)\right. \\
& \left.-\Omega\left(\mathbf{k}+\mu \frac{i}{2} \nabla_{\mathbf{X}}, \mathbf{X}_{m}-\delta \frac{i}{2} \nabla_{\mathbf{K}}\right)\right] \mathcal{E} .
\end{aligned}
$$


Here, the summation over repeated indices is understood (i.e., $\mu^{j} \partial_{T_{j}}=\sum_{j=1}^{N} \mu^{j} \partial_{T_{j}}$ ). To make the magnitude of the various terms in the operators on the right side of Eq. (11) explicit, we write the operators $\Omega$ as (see appendix A)

$$
\begin{aligned}
& \Omega\left(\mathbf{k} \mp \mu \frac{i}{2} \nabla_{\mathbf{X}}, \mathbf{X}_{m} \pm \delta \frac{i}{2} \nabla_{\mathbf{K}}\right) \\
& \quad=\left.\exp \left( \pm \beta \frac{i}{2} \nabla_{\tilde{\mathbf{X}}_{m}} \cdot \nabla_{\mathbf{K}} \mp \mu \frac{i}{2} \nabla_{\tilde{\mathbf{k}}} \cdot \nabla_{\mathbf{X}}\right) \sigma\left(\tilde{\mathbf{k}}, \tilde{\mathbf{X}}_{m}\right)\right|_{\tilde{\mathbf{k}}=\mathbf{k}, \tilde{\mathbf{X}}_{m}=\mathbf{X}},
\end{aligned}
$$

where $\beta=\varepsilon / \delta$. Physically, $\beta$ thus measures the decorrelation length scale of the waves $\left(\delta^{-1}\right)$ relative to length scale of the medium variations $\left(\varepsilon^{-1}\right)$. If $\beta \ll 1$ the wave field decorrelates over distances short relative to the bottom variations, so that regions separated by $O(1)$ medium variations are statistically independent. In fact, if $O(\mu)=O(\beta)=O(\varepsilon) \ll 1$, Eq. (11) reduces (to lowest order) to the well-known RTE (Bremmer 1972)

$$
\frac{\partial \mathcal{E}}{\partial t}+\nabla_{\mathbf{k}} \sigma \cdot \nabla_{\mathbf{x}} \mathcal{E}-\nabla_{\mathbf{x}} \sigma \cdot \nabla_{\mathbf{k}} \mathcal{E}=0
$$

where we dropped the scaling on the coordinates. From our analysis, we see that the RTE [Eq. (13)] is valid in a slowly varying medium only if the wave field decorrelates on shorter scales than the scale of the medium variations. In other words, in this limit, cross correlations induced by medium variations are lost faster than they are generated so that the wave system retains no memory of them and the wave field remains effectively homogeneous [i.e., $\mu$ remains $O(\varepsilon)$ ]. The RTE is thus valid if the spectrum of the medium variations is mostly confined to wavenumbers that are smaller than a characteristic (wavenumber) width of the wave spectrum. Because for most oceanic conditions this condition is easily satisfied, Eq. (13) is widely used and-in one form or anotherstands at the heart of most modern, large-scale, stochastic models for ocean wind waves.

However, in coastal areas exposed to ocean swells, the interaction of waves with the seafloor topography on the inner shelf (or coastal currents), or the interaction of narrowband wave field with coastal structures and headlands, can result in coherent interferences in the wave field that are visible even to a casual observer (e.g., interference in a focal zone induced by currents or topography). In such regions, the length scales of medium variations and decorrelation length scale of the waves can be of similar magnitude, so that $\beta=O(1)$, and the approximations implied in the RTE are not valid. In this case, a truncated expansion in $\beta$ is not a useful approximation, but the general transport Eq. (11) can be alternatively approximated through a Fourier integral representation of the operators as in (see appendix A)

$$
\begin{aligned}
\mu^{j} \partial_{T_{j}} \mathcal{E}\left(\mathbf{K}, \mathbf{X}, T_{1}, T_{2}, \ldots, T_{N}\right)= & -i \int d \mathbf{Q} \exp \left(i \mathbf{Q} \cdot \mathbf{X}_{m}\right)\left[\exp \left(-\frac{i}{2} \mu \nabla_{\mathbf{X}} \cdot \nabla_{\tilde{\mathbf{k}}}\right) \hat{\sigma}(\tilde{\mathbf{k}}, \mathbf{Q})\right]_{\tilde{\mathbf{k}}=\mathbf{k}} \\
& \times \mu^{j} \mathcal{E}\left(\mathbf{K}-\beta \mathbf{Q} / 2, \mathbf{X}, T_{1}, T_{2}, \ldots, T_{N}\right)+\text { C. C. }
\end{aligned}
$$

where $\mathbf{Q}=\mathbf{q} / \varepsilon$ and C.C. denotes the complex conjugate. On account of the slowly varying medium, major contributions to $\hat{\sigma}(\mathbf{k}, \mathbf{Q})$, and thus the integral, are limited to the domain $|Q| / k_{0} \leq O(1)$ so that the integral in Eq. (14) can be efficiently numerically approximated and, to $O\left(\mu^{N}\right)$, the transport Eq. (14) becomes (in physical coordinates)

$$
\partial_{t} \mathcal{E}(\mathbf{k}, \mathbf{x}, t)=-i \int_{\mathcal{D}} \hat{\Omega}^{(N)}\left(\mathbf{k}-\frac{i}{2} \nabla_{\mathbf{x}}, \mathbf{q}\right) \mathcal{E}\left(\mathbf{k}-\frac{1}{2} \mathbf{q}, \mathbf{x}\right) \exp (i \mathbf{q} \cdot \mathbf{x}) d \mathbf{q}+\text { C. C }
$$

Here $\mathcal{D}$ denotes the domain of integration such that $|\mathbf{q}| / k_{0} \leq O(\varepsilon)$, and the kernel $\hat{\Omega}^{(N)}$ operating on $\mathcal{E}$ is defined as

$$
\hat{\Omega}^{(N)}\left(\mathbf{k}-\frac{i}{2} \nabla_{\mathbf{x}}, \mathbf{q}\right)=\sum_{|n|=0}^{N} \frac{1}{n !}\left(-\frac{i}{2}\right)^{|n|} \frac{\partial^{n} \hat{\sigma}}{\partial \mathbf{k}^{n}} \frac{\partial^{n}}{\partial \mathbf{x}^{n}} .
$$

The expressions Eq. (15) and Eq. (16) describe the evolution of the second-order wave statistics while accounting for the generation and transport of coherent structures in the wave field. In this case, cross correlations can be generated (by medium variations) faster than they are destroyed, so that they can develop and persist and affect the wave statistics. Equation (16) is a central result of this paper, which we will refer to as the $N$ th-order quasi-coherent (QC) approximation, or quasi-coherent approximation if the order of the approximation is understood. The RTE [Eq. (13)] is 
(a)

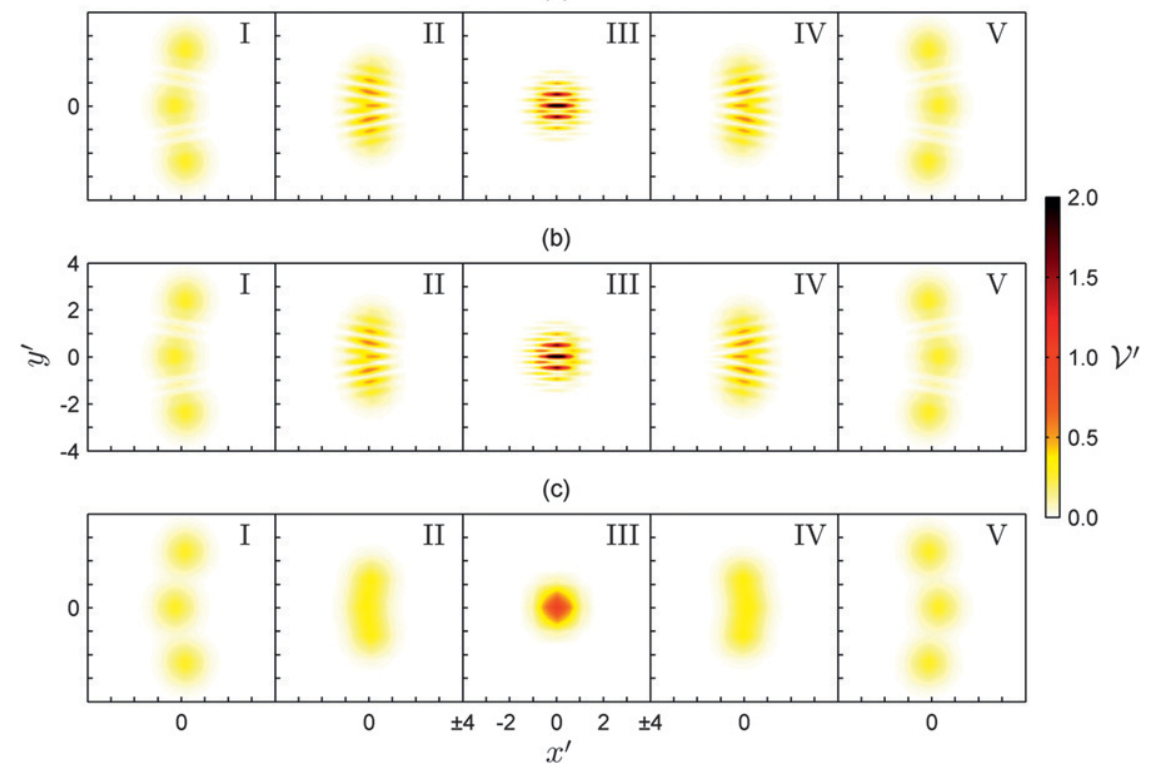

FIG. 1. Snapshots of normalized wave variance [normalization by $(3 / 2) a^{2}$ ] of three-packet interference example. (from left to right) Normalized variance is shown at discrete times $t_{I-V}$, starting at $t_{I}=110 L_{p} / v_{x}$ increasing in time intervals of $\Delta t=20 \lambda / v_{x}$. (a) Evolution for exact model, (b) the QC approximation, and (c) to the RTE. The $x^{\prime}$ and $y^{\prime}$ denote the horizontal coordinates normalized by $6 L_{p}$.

thus a special case of Eq. (15), where $O(\beta)=O(\mu)=$ $O(\varepsilon) \ll 1$.

\section{Evolution of coherent wave structures}

To illustrate the implications of the quasi-coherent approximation [Eq. (15) with Eq. (16)], and the differences with the RTE [Eq. (13)], we consider two distinct cases where cross correlations affect wave statistics. The first example considers the evolution of a group of wave packets through a homogeneous medium, where the inhomogeneity is fully determined by the initial condition and then transported through the domain. The second example considers the evolution of ocean waves over a two-dimensional topographic feature (shoal); in this case, the incident wave field is homogeneous and cross correlations are generated through the interaction with the variable medium and transported down wave of the shoal.

\section{a. Gaussian packets through a homogenous medium}

We consider a wave field consisting of three coherent Gaussian wave packets propagating in deep water, for which the surface elevation at some arbitrary initial time $(t=0)$ can be written as

$$
\zeta(\mathbf{x}, 0)=\exp \left(-\alpha|\mathbf{x}|^{2}\right) \sum_{j=1}^{3} A_{j} \exp \left(i \mathbf{k}_{j} \cdot \mathbf{x}\right)
$$

Here, the $A_{j}$ are the (complex) packet amplitudes and $\mathbf{k}_{j}$ is the carrier wavenumber. The initial spectrum (at $t=0), \hat{\mathcal{E}}(\mathbf{k}, \mathbf{u}, 0)$, is then given by

$$
\begin{aligned}
\hat{\mathcal{E}}(\mathbf{k}, \mathbf{u}, 0)= & \sum_{m=1}^{3} \sum_{n=1}^{3} \frac{\left\langle A_{m} A_{n}^{*}\right\rangle}{32 \pi^{2} \alpha^{2}} \exp \left(-\frac{1}{2 \alpha}\left|\mathbf{k}-\frac{\mathbf{k}_{m}}{2}-\frac{\mathbf{k}_{n}}{2}\right|^{2}\right. \\
& \left.-\frac{1}{8 \alpha}\left|\mathbf{u}-\mathbf{u}_{m}+\mathbf{u}_{n}\right|^{2}\right) .
\end{aligned}
$$

For a homogeneous medium, and in a reference frame that moves with the mean group velocity, the dispersion relation $\sigma(\mathbf{k})=\sqrt{g|\mathbf{k}|}+\mathbf{k} \cdot \boldsymbol{v}$, where $\boldsymbol{v}=(1 / 2) \overline{\mathbf{k}} k_{p}^{-1} \sqrt{g / k_{p}}$, and $\overline{\mathbf{k}}$ is the mean carrier wavenumber, so that Eq. (4) has the exact solution

$$
\mathcal{E}(\mathbf{k}, \mathbf{x}, t)=\int \hat{\mathcal{E}}(\mathbf{k}, \mathbf{u}, 0) \exp \left[i \mathbf{u} \cdot \mathbf{x}-i \omega_{\Delta}(\mathbf{k}, \mathbf{u}) t\right] d \mathbf{u}
$$

with

$$
\omega_{\Delta}(\mathbf{k}, \mathbf{u})=\sigma(\mathbf{k}+\mathbf{u} / 2)-\sigma(\mathbf{k}-\mathbf{u} / 2) .
$$

The relations Eqs. (19) and (20) are exact. The Nthorder QC approximation is obtained by substituting $\hat{\sigma}(\mathbf{k}, \mathbf{q})=\delta(\mathbf{q}) \sigma(\mathbf{k})$ in Eq. (15), where $\delta(\mathbf{q})$ denotes the Dirac delta function, Fourier transforming with respect to $\mathbf{x}$, and solving the resulting ordinary differential equation. On applying the inverse transform with respect to $\mathbf{u}$, 
(a)

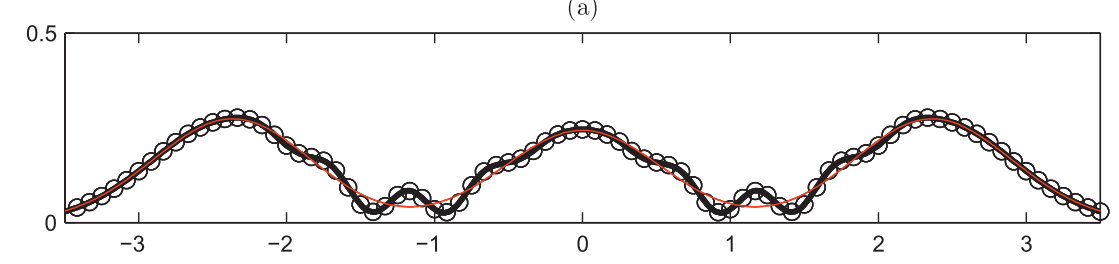

(b)

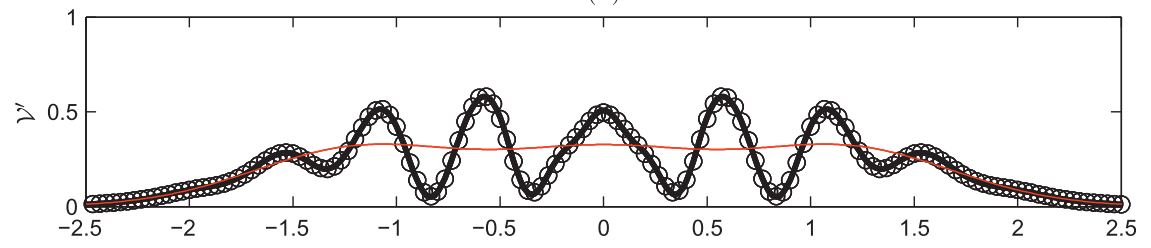

(c)

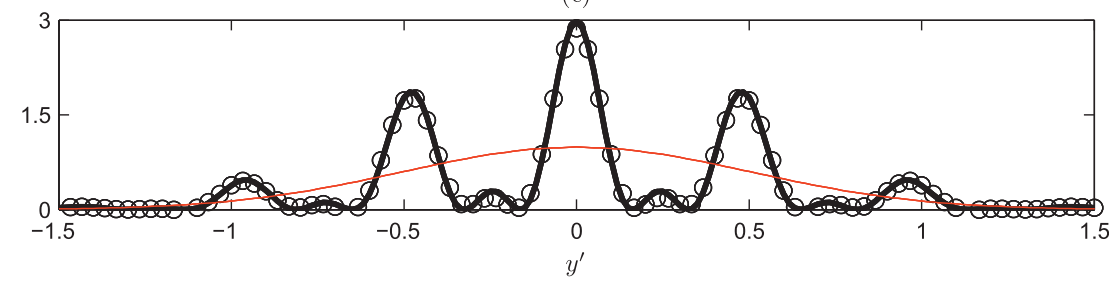

FIG. 2. Cross sections (at $x^{\prime}=0$ ) of normalized wave variance of three-packet interference example. (from left to right) The wave variance is shown at discrete times $t_{I-I I I}$, starting at (a) $t_{I}$ and increasing in time. Comparison between exact model (solid line), the QC approximation (circle markers), and the RTE (thin line). Normalization of vertical and horizontal scales and discrete times as in Fig. 1. Note that the horizontal and vertical range can vary among panels.

the result is again Eq. (19), but with $\omega_{\Delta}$ replaced by its $N$ th-order Taylor series in $\mathbf{u}$ around $\mathbf{u}=0$,

$$
\omega_{\Delta}^{(N)}(\mathbf{k}, \mathbf{u})=\sum_{|n|=1,3, \ldots}^{|n| \leq N} \frac{\mathbf{u}^{n}}{n ! 2^{|n|-1}} \frac{\partial^{n} \sigma}{\partial \mathbf{k}^{n}}
$$

In what follows, we consider three coherent packets of the same energy and carrier wavelength $\left(\left|A_{j}\right|=a,\left|\mathbf{k}_{j}\right|=k\right)$, that propagate at angles of $-20^{\circ}, 0^{\circ}$, and $20^{\circ}$ relative to the positive $x$ axis. We set $\alpha=(1 / 36) L_{p}^{-2}$, so that a characteristic length scale of the packets is roughly six wavelengths $(\mu \approx 1 / 6)$, and we consider the evolution from $t=-150 T_{p}$ to $t=20 T_{p}$ (with $T_{p}=L_{p} / v_{x}$ a characteristic period). The QC approximation is initialized at $t=-150 T_{p}$ using the exact solution.

Because the simulation time is roughly $T_{p} / \mu^{3}$, we use a third-order QC approximation [by truncating Eq. (21) after the second term]. Each packet is calculated individually on a discrete equidistant $\mathbf{k}$-mesh centered at $\mathbf{k}_{m n}$ using 30 points in each direction with a mesh size of $\Delta k=\sqrt{\alpha} / 5$. The Fourier integrals are approximated using a fast Fourier transform with $u_{1}$ and $u_{2}$ discretized as $(-31 \ldots 31) \Delta u$, with $\Delta u=\sqrt{\alpha} / 5$, and the result interpolated to a discrete $241 \times 241 \mathbf{x}$-mesh centered at the origin with mesh size $\Delta x=\Delta y=L_{p} / 5$.
The initial evolution of the wave system is characterized by convergence and interference of the wave packets (Fig. 1a), followed by defocusing and divergence, after which the packets emerge unchanged and the initial state is recovered. The QC approximation captures the principal dynamics of the wave evolution, including the coherent interference (Figs. 1b and 2), which confirms that the QC approximation accurately represents crosscorrelations in the evolution of random waves associated with the coherent interference of wave packets traveling at moderate angles. In contrast, the wave packet evolution as represented by the RTE (Figs. 1c and 2) is dramatically different from the exact result; in particular, the interference pattern at $t=t_{I I I}$ is not resolved because the RTE does not account for the transport of cross-variance contributions $(m \neq n)$.

The differences in evolution are apparent also from the spectra (left panels in Fig. 3). The variance density spectrum at $x^{\prime}=0$ (where $\mathbf{x}^{\prime}=\mathbf{x} / L_{p}$ ) contains a single positive peak for $t_{I}$ and $t_{I I I}$ related to the central packet, and three peaks at $t_{I I I}$ when the three packets have converged (Fig. 3a). Because the CM spectrum accounts for intermode coupling, it contains additional interference peaks, which travel along rays midway between the rays of the autovariance contributions involved in the interference. For the case considered here, at $x^{\prime}=0$, the 
(a) auto-contributions

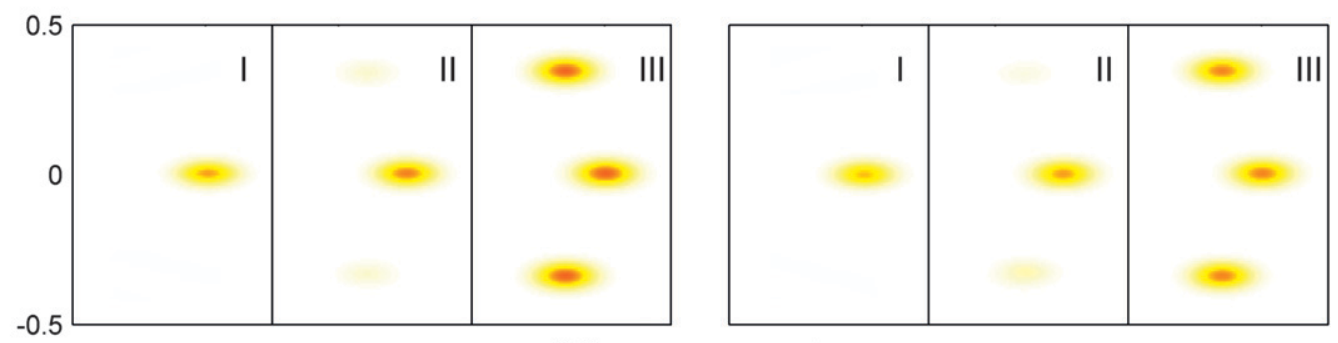

(b) cross-contributions
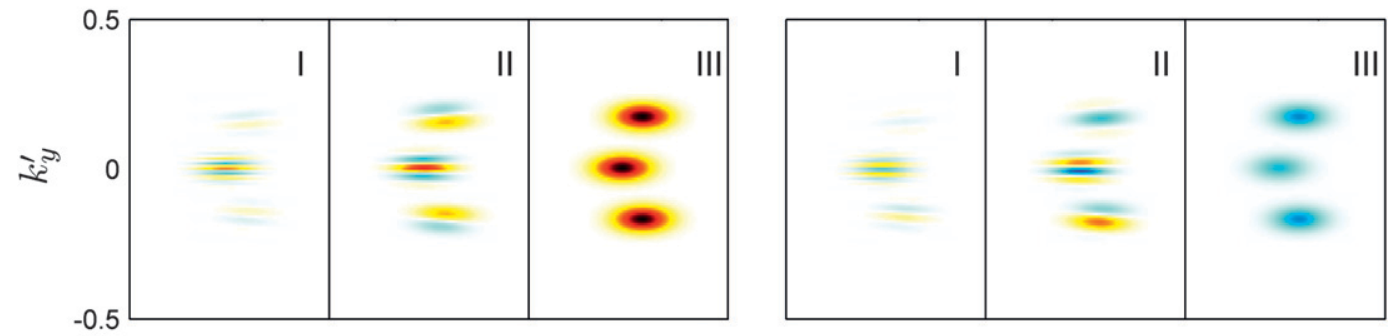

(c) CM spectrum

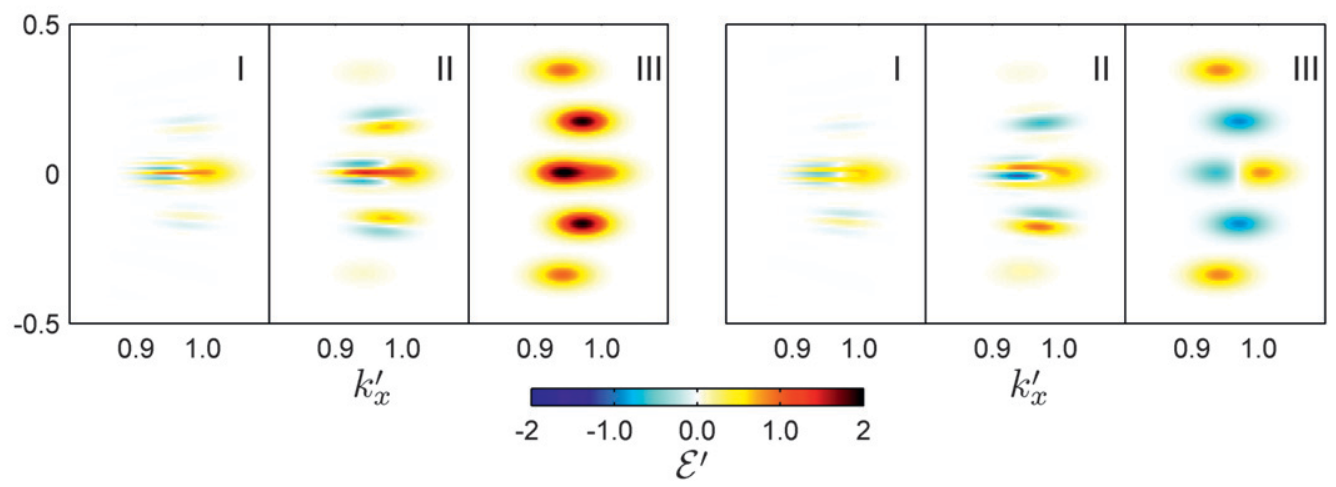

FIG. 3. Spectral evolution of the three-packet interference example Eq. (18) evaluated at (left) $\left(x^{\prime}, y^{\prime}\right)=0$ and (right) $\left(x^{\prime}, y^{\prime}\right)=(0,1 / 6)$ for $t_{I-I I I}$ (times as defined in caption Fig. 1). (a) The autovariance contributions, (b) the crossvariance contributions, and (c) the resulting CM spectrum (sum of auto- and cross-variance contributions) are shown. The spectral coordinates $k_{x}^{\prime}$ and $k_{y}^{\prime}$ are normalized with $k_{p}$ and spectra are normalized with the peak contribution of an individual packet $(4 \pi \alpha)^{-1}$.

cross variance at $t_{I}$ consists of a single interference peak (Fig. 3b), which represents the coherence between the outer packets, and travels along the ray $y^{\prime}=0$. Coherence between the center and outer packets travels along different rays and only manifests itself at $t_{I I}$ and $t_{I I I}$ where these rays cross through $y^{\prime}=0$. The CM spectrum is the sum of the interference contributions and (auto)variance density spectrum (Fig. 3c). The spectral interference terms capture the rapid spatial oscillations of the wave statistics as a result of wave interference in the focal zone (Fig. 1c). This fast-scale variability is also seen in the spectral domain, when the spectra are evaluated at a slightly offset location (cf. left and right panels in Fig. 3). Note that the wave packets in this example do not "interact" with each other in the usual sense, and the coherent interference is completely determined a priori by the inclusion of interference peaks in the initial condition. No cross correlations are generated (or destroyed) in the course of the evolution.

\section{b. Coherent interference patterns induced by topography}

To consider the generation and persistence of coherent interference patterns through the interaction with a slowly varying medium, we compare model simulations with laboratory observations of waves traveling over a submerged shoal (Vincent and Briggs 1989). In these experiments, an elliptic shoal with its crest $15.24 \mathrm{~cm}$ below still-water level was placed in a wave basin with a uniform depth of $h=45.72 \mathrm{~cm}$ (see Fig. 4). 


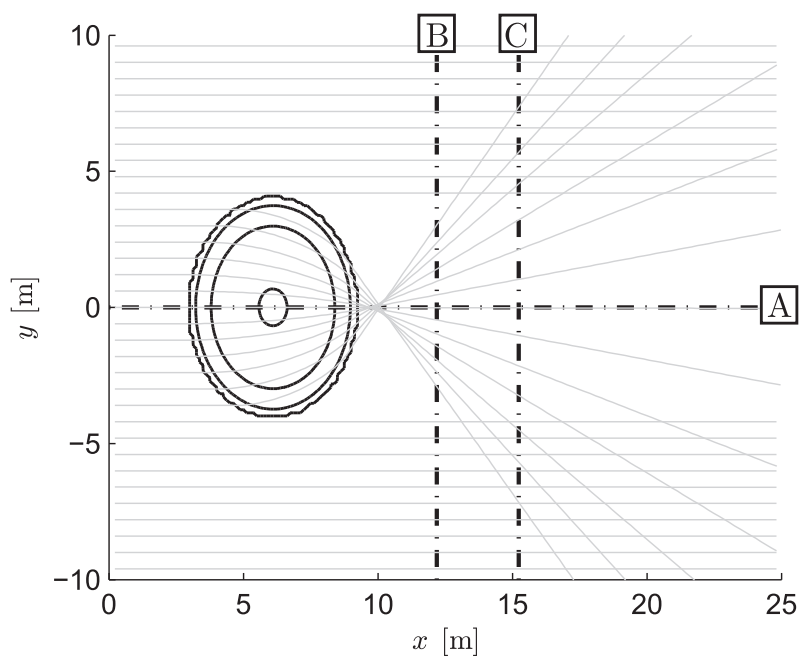

FIG. 4. Plan view of the experimental setup by Vincent and Briggs (1989) including a ray-traced solution (thin gray lines) for unidirectional monochromatic waves (period $1.3 \mathrm{~s}$, incident direction $\theta_{0}=$ $0^{\circ}$ ). Depth contours (drawn for $0.15-0.45 \mathrm{~m}$, at 0.1 -m intervals) are indicated by black solid lines; instrumented transects (at $y=0 \mathrm{~m}, x=$ $12.19 \mathrm{~m}$, and $x=15.24 \mathrm{~m}$ ) are indicated with dash-dot lines.

We consider a case with monochromatic waves (M2) and random waves with a TMA spectrum (N4); for the latter, the peak enhancement factor $\gamma=20$, directional spreading is approximately $10^{\circ}$ [as defined by Kuik et al. (1988)], and the (significant) wave height and (peak) period are $2.54 \mathrm{~cm}$ and $1.3 \mathrm{~s}$, respectively. For additional details we refer to Vincent and Briggs (1989).

We compare model simulations with a first-order QC model and the RTE to the laboratory observations. Because the observational data is sparse, we also include a comparison with simulations with the deterministic hydrodynamical model Simulating Waves Till Shore (SWASH), which has been independently validated (see Zijlema et al. 2011).

The spectral models are numerically evaluated on a rectangular spatial $(30 \mathrm{~m} \times 25 \mathrm{~m})$ and spectral domain $\left[4 k_{p} \times 3 k_{p}\right.$, starting at $\left.\mathbf{k}=\left(-0.5 k_{p},-1.5 k_{p}\right)\right]$, uniformly discretized with mesh sizes $\Delta x=\Delta y=12.5 \mathrm{~cm}$ and $\Delta \mathbf{k} / k_{p}=(18,20)$. We use second-order upwind finitedifference approximations for the spatial (and spectral) gradients, in combination with an explicit first-order time stepping. The integral in Eq. (15) is approximated by a second-order numerical quadrature on the domain $\mathcal{D}$ delimited by $|\mathbf{q}|<2 k_{p}$. At the wave maker, the incident spectrum is imposed, and periodicity is assumed in the lateral direction. Combined with a radiation-type boundary condition opposite to the wave maker, the solution is then marched in time until a steady state is reached.

Over the shoal, the incident monochromatic waves (case M2) are refracted in different directions, resulting in fast lateral variations in wave variance behind the shoal, because of the coherent interference of the crossing wave components. The QC model captures the variations in wave energy induced by these interferences and agrees well with observations and the deterministic model [Eq. (5)]. In contrast, the RTE predicts much stronger focusing and lower wave heights in the shadow zone (see Fig. 5), and does not resolve the finescale structure in wave energy associated with wave coherency.

For the random incident wave field (case N4), because of the increased dispersion (mostly directional), the wave field decorrelates faster, resulting in a smoothing of the wave statistics behind the shoal (see Fig. 6). In this case, the QC results are qualitatively more similar to the RTE result, although several differences remain. In the region directly behind the shoal, wave heights predicted by QC theory are consistent with observations and those obtained with the deterministic model, whereas the wave heights predicted by the RTE are approximately $20 \%$ lower. If the directionality (and thus dispersion) is reduced, say to $3^{\circ}\left(\mathrm{N}^{\prime}\right)$ a well-defined interference pattern emerges again and the QC model provides a much more realistic presentation of the wave statistics than the RTE. ${ }^{3}$ These cases, which can represent the propagation of narrowband swell waves over coastal topography, show that including coherent effects can be significant for situations that are of practical interest. The emergence and persistence of coherent interferences in narrowband random waves over coastal topography can be important for regional wave statistics and thus affect wave-driven circulation and transport processes in such areas.

\section{Discussion}

Coherent wave interference patterns are common in the coastal ocean, for instance because of the interaction with submerged topography, currents, islands, headlands, or coastal structures. Statistical models based on the RTE do not account for such interference patterns, and this can result in systematic differences between observed variations in wave statistics and model predictions. In this work we introduce a new transport model that we refer to as a CM spectrum, which includes the effects of coherent interference on the wave statistics. The concept of a CM spectrum to describe the statistical evolution of inhomogeneous random processes is not new, but it has been developed independently across various fields, such as the Wigner distribution in quantum mechanics and optics (e.g., Bastiaans 1997; Bremmer 1972; Wigner 1932), the concept of generalized radiance

\footnotetext{
${ }^{3}$ This case was not considered by Vincent and Briggs.
} 

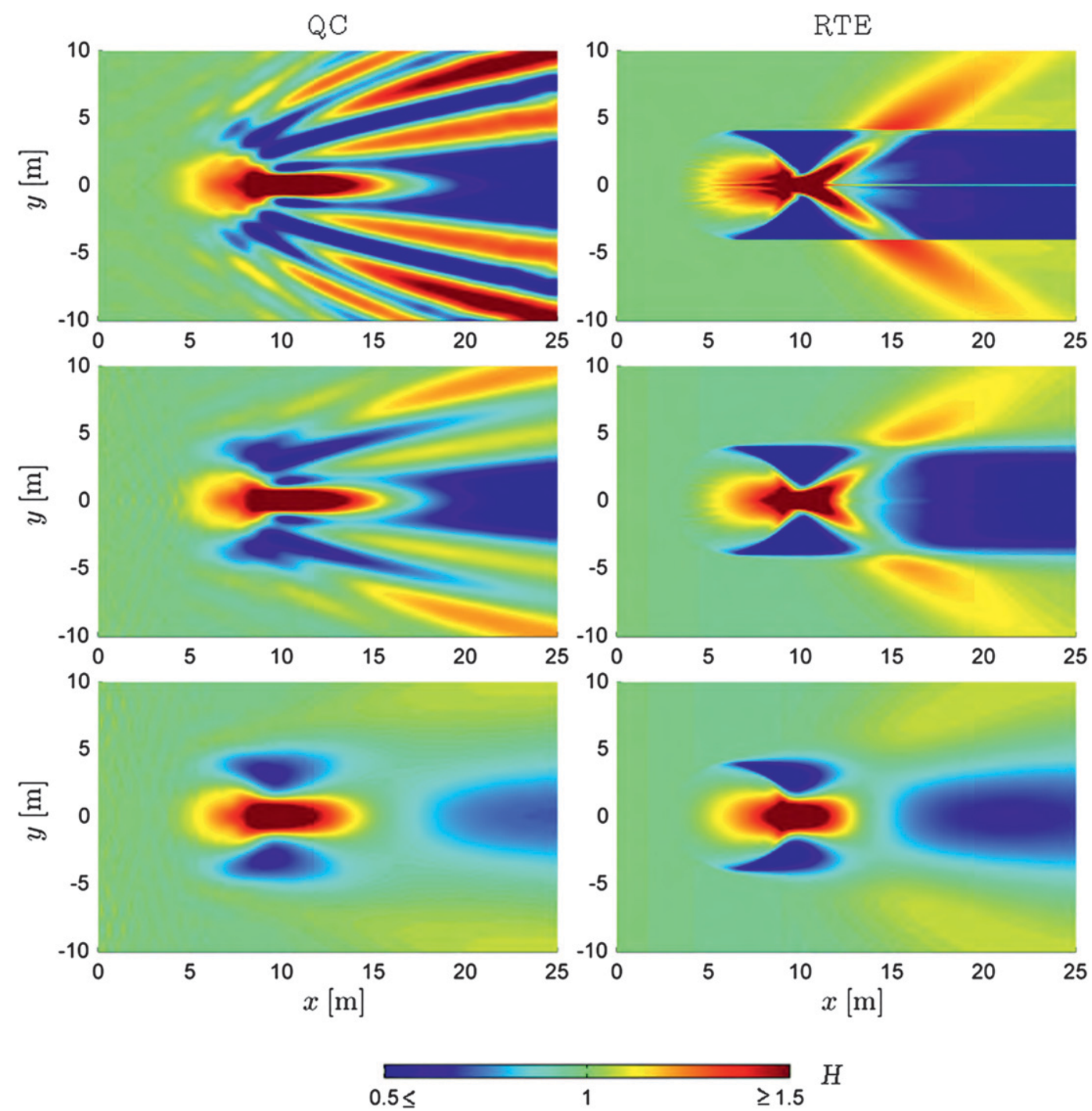

FIG. 5. Plan view of modeled (normalized) wave heights for the experimental setup as considered by Vincent and Briggs (1989) for (top) case M2, (bottom) case N4, and (middle) the additional case N4' considered in the present work. Comparison between (left) the $\mathrm{QC}_{1}$ approximation and (right) the $\mathrm{RTE}$ shows that the $\mathrm{QC}_{1}$ approximation, in contrast to the RTE, resolves the finescale interference pattern in the focal zone of a topographical lens.

in radiometry (Walther 1968), and the Wigner-Ville distribution in signal processing (Ville 1948; Cohen 1989). Here we apply these ideas to ocean waves traveling through a variable medium and in the presence of caustics, such as are commonly found in, for example, coastal areas and other regions characterized by relatively strong two-dimensional medium variations.

\section{a. Interference terms}

Apart from energy (or variance) contributions, the coupled-mode spectrum carries cross-correlation and cross-phase information on coherent interferences between noncolinear wave components in the wave field.
For example, if we revisit the correlated wave packet example in section 3, and-for convenience-reduce it to two packets propagating at some equal but opposite angle with respect to the positive $x$ axis, so that $\mathbf{k}_{1}=(\varkappa, \lambda)$, $\mathbf{k}_{2}=(\varkappa,-\lambda)$, and $A_{j}=a_{j} \exp i \phi_{j}$, with $a_{j}=\left|A_{j}\right|$ and $\phi_{j}=$ $\arg A_{j}$, the CM spectrum can be written as

$$
\begin{aligned}
\mathcal{E}(\mathbf{k}, \mathbf{x})= & \frac{1}{2} \int \hat{\mathcal{E}}(\mathbf{k}, \mathbf{u}) \exp (i \mathbf{k} \cdot \mathbf{u}) d \mathbf{u} \\
= & \frac{e^{-2 \alpha|x|^{2}}}{4 \pi \alpha}\left[\left\langle a_{1}^{2}\right\rangle G_{11}+\left\langle a_{2}^{2}\right\rangle G_{22}\right. \\
& \left.+2\left\langle a_{1} a_{2}\right\rangle G_{12} \cos \left(2 \lambda y+\left\langle\phi_{1}-\phi_{2}\right\rangle\right)\right],
\end{aligned}
$$



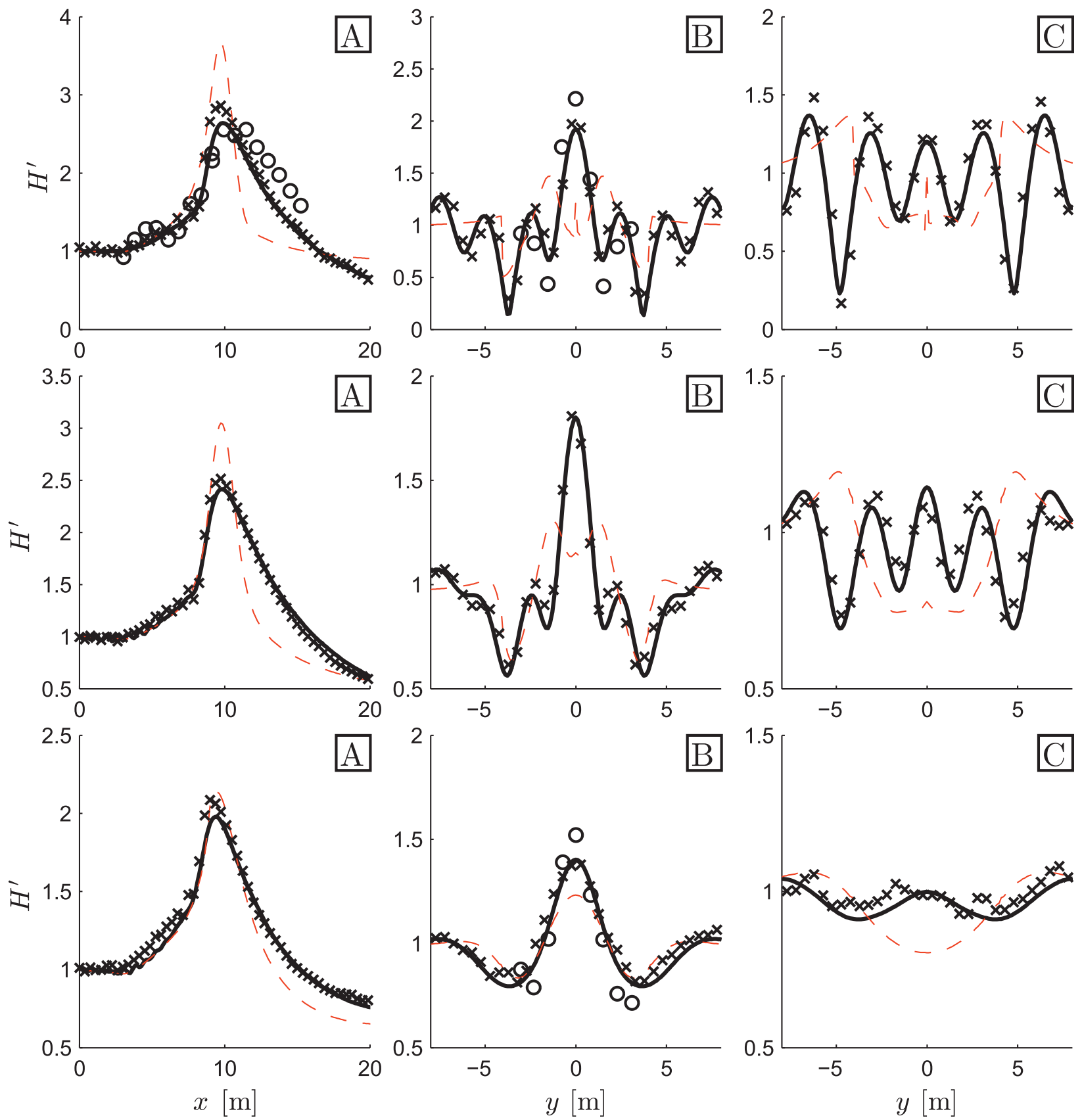

FIG. 6. Shown are normalized wave heights along transects (a) across and (b),(c) behind the shoal (see Fig. 4 for locations) as considered by Vincent and Briggs (1989) for (top) case M2, (bottom) case N4, and (middle) the additional case N4' considered in the present work. Comparison is between the QC approximation (solid black line), observations (circle markers, when available), the deterministic model SWASH (crosses), and the RTE (dashed red line).

where the $\hat{\mathcal{E}}(\mathbf{k}, \mathbf{u})$ is the same as in Eq. (18) and $G_{i j}=\exp \left[(1 / 2 \alpha)\left|\mathbf{k}-\left(\mathbf{k}_{i} / 2\right)-\left(\mathbf{k}_{j} / 2\right)\right|^{2}\right]$. The last term in Eq. (22) is a coupled-mode contribution that represents the contribution from the coherent interference between the two packets. This contribution is located midway between the associated autovariance contributions (Hlawatsch and Flandrin 1997), can become negative and, because it does not directly correspond to a field component, does not carry energy itself.

Instead, this contribution determines how the energy of the wave field is distributed between kinetic and potential energy. After all, in a statistically homogeneous (and linear) wave field, the energy is equipartitioned between potential and kinetic energy so that knowledge 
of either potential or kinetic energy suffices. On the other hand, in a wave field that undergoes coherent interference, information on the distribution of potential and kinetic energy is required to fully characterize the wave field statistics. This information is provided by the cross contributions in the coupled-mode spectrum. In our example [Eq. (22)], the interference results in a coherent standing wave motion along the $y$ coordinate where the wave packets alternately interfere constructively and destructively; the coupled mode thus contributes negatively in the nodes (where more energy is kinetic) and positively in the antinodes (where more energy is potential).

\section{b. Aperture limitation on wave interferences}

The CM spectrum is a general representation of the complete second-order statistics, including interferences between wave component with arbitrary mutual orientation, and without inherent aperture limitation. However, the QC transport equation, which is based on a series expansion of $\hat{\sigma}[\mathbf{k} \pm(\mathbf{u} / 2), \mathbf{q}]$ in $\mathbf{u}$ [Eq. (A6)], is fundamentally more restricted, and cannot accurately transport cross correlations between waves that enclose an angle greater than $\pi / 2$. This limitation can be understood from the fact that the kernel $\hat{\Omega}^{(N)}$ [Eq. (16)] is obtained by Fourier transforming (with respect to $\mathbf{u}$ ) the series expansion of $\hat{\sigma}[\mathbf{k} \pm(\mathbf{u} / 2), \mathbf{q}]$ [Eq. (A6)]. Before truncation (for $N \rightarrow \infty$ ), this is exact if the series approximation to $\hat{\sigma}[\mathbf{k} \pm(\mathbf{u} / 2), \mathbf{q}]$ converges $\forall \mathbf{u}$. However, the dispersion relation for ocean waves depends on $|\mathbf{k}|$, so that $\nabla_{\mathbf{k}} \sigma$ is singular at $\mathbf{k}=0$, and the radius of convergence of the series expansion for $\sigma(|\mathbf{k}|)$ around $\mathbf{k}_{0}$ is $\left|\mathbf{k}_{0}\right|$. As a consequence, the operators are generally approximations and, because in the QC approximation the expansions are in $(1 / 2) \mathbf{u}$, the maximum enclosed angle is $\pi / 2\left[\mathbf{k}^{+} \cdot \mathbf{k}^{-}=0, \mathbf{k}^{ \pm}=\mathbf{k} \pm(\mathbf{u} / 2)\right]$, when both wave components are on the circle $|\mathbf{u}|=2 k$ (see Fig. 7). Therefore, if $\mathbf{k}_{0}$ is a typical wavenumber, the smallest spatial scale that can be resolved by the QC approximation is limited to $\pi / k_{0}$. Such aperture restrictions only apply to the coherent interference terms; the spectrum of the automode distributions (variance-carrying contributions) is arbitrary.

\section{c. Wide-angle diffraction}

The propagation of ocean waves around thin barriers and obstructions results in coherent interferences, associated with diffraction. From a statistical viewpoint, such diffraction patterns are a coherent interference pattern originating from the interaction with the barrier. Although these effects are ignored by the RTE, they can be readily accounted for in the QC approximation.

To illustrate this, we apply the QC approximation to the propagation of waves through a gap (extending over

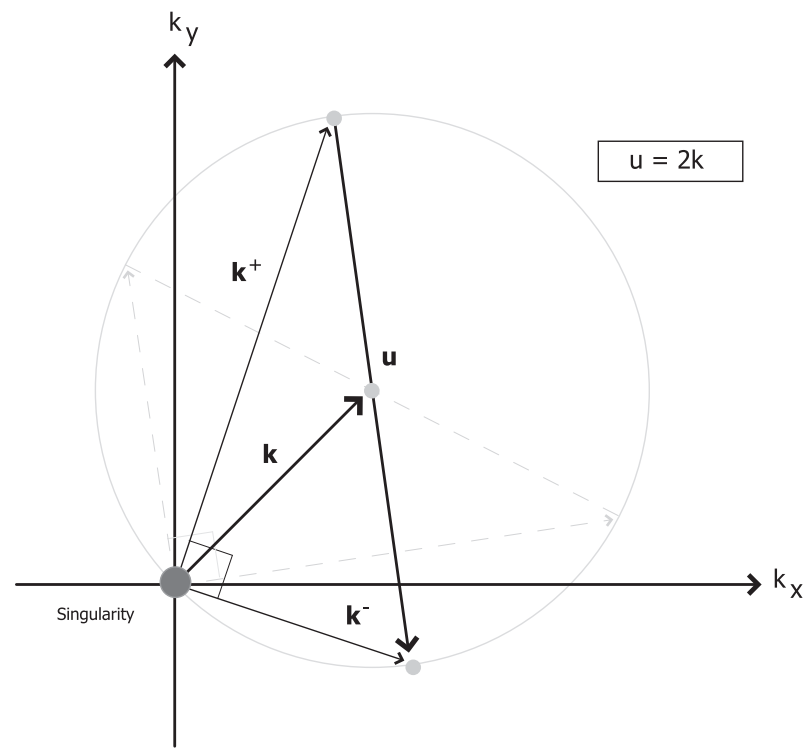

FIG. 7. Sketch of wave interference geometry on the radius of convergence. For a dispersion relation $\Omega(|\mathbf{k}|)$ (where $|\mathbf{k}|$ is singular at the origin, as indicated in the figure), the radius of convergence of the QC approximation (for a particular $\mathbf{k}$ ) is given by $|\tilde{\mathbf{u}}|=2 k$. Consequently, components $\left(\mathbf{k}^{+}, \mathbf{k}^{-}\right)$with average wavenumber $\mathbf{k}=$ $\left(\mathbf{k}^{+}+\mathbf{k}^{-}\right) / 2$, which are located on the limit circle $\left(\left|\mathbf{k}^{+}-\mathbf{k}^{-}\right|=\right.$ $2|\mathbf{k}|$ ), propagate perpendicular to each other, as shown in the figure (solid black and striped gray lines).

$-G_{1}<y<G_{2}$ ), in an otherwise rigid, but fully absorbing barrier (situated at $x=0$ ). Apart from the obstacle, the medium is uniform and the dispersion relation of the form $\sigma=\sigma(|\mathbf{k}|)$. The incident wave field is homogeneous, unidirectional, and normally incident on the barrier with a known variance density spectrum $\mathcal{S}_{0}(\omega)$. Using the Kirchhoff, or physical optics, approximation (see appendix C), and a third-order QC approximation, we obtain the $\mathcal{E}$ spectrum just behind the barrier $\left(x=0^{+}\right), \mathcal{E}_{0}(\mathbf{k}, y)$.

Behind the barrier, the coupled-mode spectrum is given by Eq. (19), and for a stationary solution we have $\omega_{\Delta}(\mathbf{k}, \mathbf{u})=\sigma(|\mathbf{k}+\mathbf{u} / 2|)-\sigma(|\mathbf{k}-\mathbf{u} / 2|)=0$ [see Eq. (20)], so that $|\mathbf{k}+\mathbf{u} / 2|=|\mathbf{k}-\mathbf{u} / 2|$ and $\mathbf{k} \cdot \mathbf{u}=0$. Therefore we have $\hat{\mathcal{E}}(\mathbf{k}, \mathbf{u})=\hat{\mathcal{E}}\left(\mathbf{k}, u_{y}\right) \delta\left(u_{x}+u_{y} k_{y} k_{x}^{-1}\right)$ for $k_{x}>0$ (and zero elsewhere), and [from Eq. (19)] find that

$\mathcal{E}(\mathbf{k}, \mathbf{x})=\int \hat{\mathcal{E}}\left(\mathbf{k}, u_{y}\right) \exp \left(i u_{y} y-i u_{y} k_{y} k_{x}^{-1} x\right) d u_{y}$.

The wave statistics for $x>0$, including coherent interferences associated with diffraction, are thus entirely determined from the boundary condition $\hat{\mathcal{E}}\left(\mathbf{k}, u_{y}\right)$ at $x=$ 0 (the gap). No new information is added as the waves travel behind the barrier, and the diffraction effects commonly seen in harbors or from aerial photography of waves around islands, are-from a statistical point 

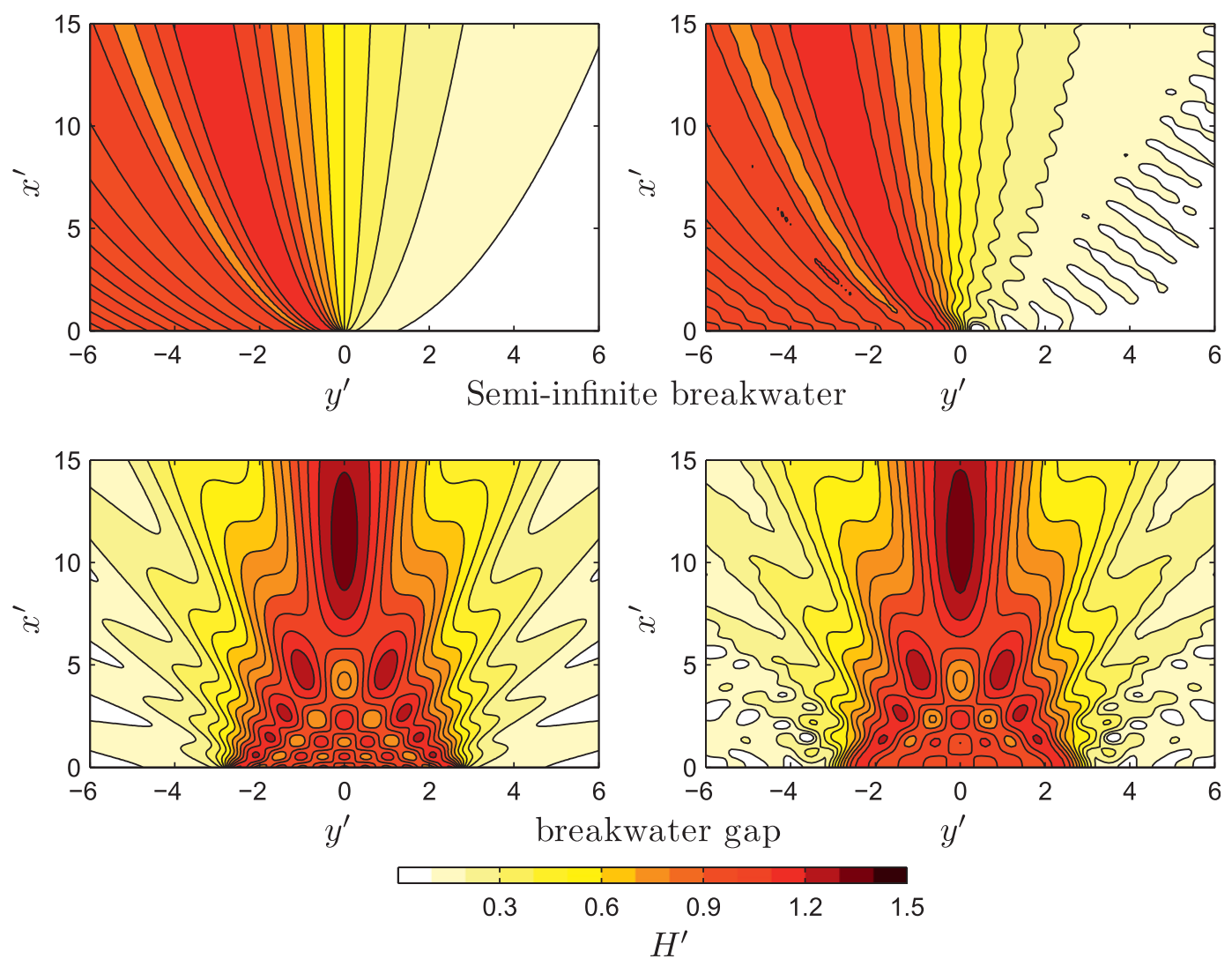

FIG. 8. Contours of normalized wave height $H^{\prime}=\sqrt{\mathcal{V} / \mathcal{V}_{0}}$ (where $\mathcal{V}_{0}$ is the variance of the incident waves) behind (top) a semi-infinite breakwater and (bottom) a breakwater gap. The (left) analytic solution (Penney and Price 1952) and (right) QC approximation are shown. The incident wave field consists of unidirectional waves, normally incident onto the breakwater, with a peak angular frequency of $\omega_{p}=\pi \mathrm{rad} / s, k_{p} h=1.2$ and a narrowband Gaussian-shaped frequency distribution with characteristic width of $\Delta \omega=(1 / 100) \omega_{p}$. The horizontal coordinates $\left(x^{\prime}, y^{\prime}\right)$ are normalized with the peak wavelength.

of view-simply the manifestation of cross correlations determined by the up-wave boundary conditions, transported by Eq. (23).

In Figs. 8 and 9 we compare simulation results with analytic solutions (Penney and Price 1952) for a monochromatic incident wave field impinging on a semiinfinite breakwater $\left(G_{1}=L_{y} / 2\right.$ and $G_{2}=0$, with $L_{y}$ the lateral extent of the domain), and a barrier gap $\left(G_{1}=\right.$ $\left.G_{2}=2.65 L_{p} \ll L_{y}\right)$. The QC approximation is in excellent agreement with the analytic solution for distances greater than about 4 wavelengths behind the barrier $\left(x / L_{p}>4\right)$, which confirms the accuracy of this approach in the intermediate to far field. Differences in the near field are principally due to the omission of evanescent modes (especially in the region $x / L_{p}<1$ ) and the use of an approximate (Kirchhoff) boundary condition (see, e.g., Stamnes 1986). These examples illustrate that the QC approximation, despite its fundamental $\pi / 2$ aperture restriction on interference terms, can accurately represent wide-angle coherencies resulting from diffraction, as implied by the good agreement with the analytic solutions.

\section{d. Relation to other transport equations}

In the derivation of the stochastic model Eq. (4) we started, following Bremmer (1972), from the wave Eq. (3). This approach is quite general and makes no explicit assumptions regarding the bandwidth of the wave field. Alternatively, if we restrict our derivation to narrowband waves from the outset, we could, following Wigner (1932) and Alber (1978), start with specific evolution equations for a narrowband wave train. To show that our approach and resulting transport equations are indeed consistent with earlier results for narrowband waves, we consider the free-surface elevation $\zeta$ of a zero-mean, narrowband wave field in deep water, given by $\zeta(\mathbf{x}, t)=A(\mathbf{x}, t) \exp \left(i \mathbf{k}_{0} \cdot \mathbf{x}-i \omega_{0} t\right)$, where $\mathbf{k}_{0}=$ $\left(\mathbf{k}_{0}, 0\right)^{\mathrm{T}}$ is the principal wavenumber, $\omega_{0}=\sigma\left(k_{0}\right)$ the angular frequency, and $A(\mathbf{x}, t)$ denotes the slowly changing wave envelope. Assuming that the bandwidth $\Delta k / k_{0}$ is 

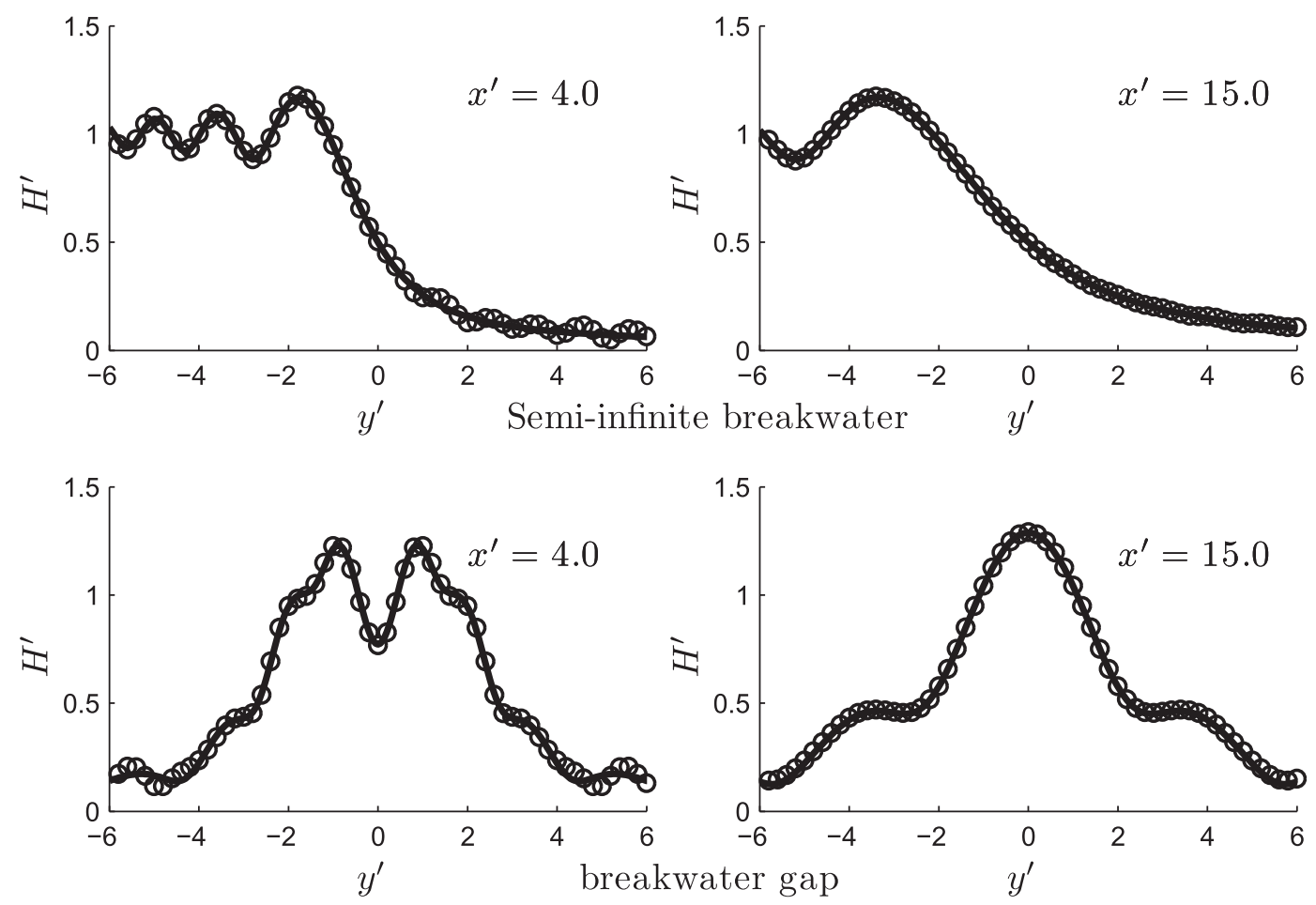

FIG. 9. Cross sections of normalized wave height $H^{\prime}=\sqrt{\mathcal{V} / \mathcal{V}_{0}}$ (where $\mathcal{V}_{0}$ is the variance of the incident waves) along (left) $x^{\prime}=4$ and (right) $x^{\prime}=15$ and behind (top) a semi-infinite breakwater and (bottom) a breakwater gap. Shown are the analytic solution [solid line from Penney and Price (1952)] and the QC approximation (circle markers). Incident wave field and coordinates as in Fig. 8.

$O(\delta)$ and the steepness is small $\left(A_{0} k_{0} \ll \delta\right.$, with $A_{0}$ a typical wave amplitude), the linear evolution of the envelope, up to $O\left(\delta^{3}\right)$, is governed by the linear part of the Dysthe equation (Dysthe 1979)

$$
\begin{aligned}
& \left\{\frac{\partial}{\partial t}+\sigma_{k_{0}}^{(1)} \frac{\partial}{\partial x}-\frac{i}{2} \sigma_{k_{0}}^{(2)} \frac{\partial^{2}}{\partial x^{2}}-\frac{i}{2 k_{0}} \sigma_{k_{0}}^{(1)} \frac{\partial^{2}}{\partial y^{2}}\right. \\
& \left.-\frac{1}{2 k_{0}^{2}}\left[k_{0} \sigma_{k_{0}}^{(2)}-\sigma_{k_{0}}^{(1)}\right] \frac{\partial^{3}}{\partial x \partial y^{2}}-\frac{1}{6} \sigma_{k_{0}}^{(3)} \frac{\partial^{3}}{\partial x^{3}}\right\} A=0 .
\end{aligned}
$$

The procedure for obtaining the evolution equations for the CM spectrum from the governing Eq. (24) is essentially the same as that followed in section 2, and equivalent to the procedure followed by Alber (1978) and Cohen (2010). We evaluate Eq. (24) at two different locations for $A_{1}=A\left(\boldsymbol{x}_{1}, t\right)$ and $A_{2}^{*}=A^{*}\left(\mathbf{x}_{2}, t\right)$; we multiply the first equation with $A_{2}^{*}$, the second with $A_{1}$, sum the two resulting equations, and ensemble average the result. After introducing the spatial average and difference coordinates $\mathbf{x}=\left(\mathbf{x}_{1}+\mathbf{x}_{2}\right) / 2$ and $\boldsymbol{\xi}=\mathbf{x}_{1}-\mathbf{x}_{2}$, we obtain an evolution equation for the product $\rho=(1 / 2)\left\langle A_{1} A_{2}^{*}\right\rangle$. Upon applying the Fourier transform with respect to $\xi$, we can express (24) in terms of the $\mathrm{CM}$ spectrum

$$
\begin{aligned}
& \left\{\frac{\partial}{\partial t}+\left[\sigma_{k_{0}}^{(1)}+\left(k_{x}-k_{0}\right) \sigma_{k_{0}}^{(2)}\right] \frac{\partial}{\partial x}+\frac{k_{y}}{k_{0}} \sigma_{k_{0}}^{(1)} \frac{\partial}{\partial y}-\frac{1}{24} \sigma_{k_{0}}^{(3)} \frac{\partial^{3}}{\partial x^{3}}\right. \\
& +\frac{1}{2}\left(k_{x}-k_{0}\right)^{2} \sigma_{k_{0}}^{(3)} \frac{\partial}{\partial x}-\frac{1}{k_{0}^{2}}\left[k_{0} \sigma_{k_{0}}^{(2)}-\sigma_{k_{0}}^{(1)}\right] \\
& \left.\quad \times\left[\frac{1}{8} \frac{\partial^{3}}{\partial x \partial y^{2}}-\frac{k_{y}^{2}}{2} \frac{\partial}{\partial x}-\left(k_{x}-k_{0}\right) k_{y} \frac{\partial}{\partial y}\right]\right\} \mathcal{E}=0 .
\end{aligned}
$$

where we used that $\mathcal{E}(\mathbf{k}, \mathbf{x})=\hat{\rho}\left(\mathbf{k}-\mathbf{k}_{0}, \mathbf{x}\right)$, with $\hat{\rho}$ denoting the transform of $\rho$ with respect to $\xi$. The first three terms inside the brackets on the left side of Eq. (25) correspond to the linear spectral evolution equation of Alber (1978). Moreover, Eq. (25) is a narrowband approximation (around $\mathbf{k}_{0}$ ) of the third-order QC approximation in a uniform medium. This can be seen if we consider the Taylor approximations for $\hat{\Omega}^{(3)}\left[\mathbf{k} \mp(i / 2) \boldsymbol{\nabla}_{\mathbf{x}}, \mathbf{q}\right]$ in $\mathbf{k}$ around $\mathbf{k}_{0}$ which, when retaining terms up to $O\left(\delta^{3}\right)$, reads 


$$
\begin{aligned}
\hat{\Omega}^{(3)}\left(\mathbf{k} \mp \frac{i}{2} \nabla_{\mathbf{x}}, \mathbf{q}\right)= & \mp \frac{i}{2} \delta(\mathbf{q})\left(\left\{\sigma_{k_{0}}^{(1)}+\left(k_{x}-k_{0}\right) \sigma_{k_{0}}^{(2)}+\frac{1}{2}\left(k_{x}-k_{0}\right)^{2} \sigma_{k_{0}}^{(3)}+\frac{k_{y}^{2}}{2 k_{0}^{2}}\left[k_{0} \sigma_{k_{0}}^{(2)}-\sigma_{k_{0}}^{(1)}\right]\right\} \frac{\partial}{\partial x}\right. \\
& \left.+\left\{\frac{k_{y}}{k_{0}} \sigma_{k_{0}}^{(1)}+\frac{\left(k_{x}-k_{0}\right) k_{y}}{k_{0}^{2}}\left[k_{0} \sigma_{k_{0}}^{(2)}-\sigma_{k_{0}}^{(1)}\right]\right\} \frac{\partial}{\partial y}-\frac{\sigma_{k_{0}}^{(3)}}{24} \frac{\partial^{3}}{\partial x^{3}}-\frac{1}{8 k_{0}^{2}}\left[k_{0} \sigma_{k_{0}}^{(2)}-\sigma_{k_{0}}^{(1)}\right] \frac{\partial^{3}}{\partial x \partial y^{2}}\right) .
\end{aligned}
$$

If the expression Eq. (26) is substituted in the transport Eq. (15), Eq. (25) follows, which shows that our results are consistent with earlier results for narrowband wave fields and contain the linear part of the Alber equation as a special case.

\section{Conclusions}

We have presented a new transport model for the statistics of inhomogeneous wave fields of arbitrary bandwidth propagating through a variable medium. The model accounts for the generation and transport of coherent interferences between wave components that enclose angles smaller than $\pi / 2$ radians. The theoretical framework presented here is a natural extension of the radiative transfer equation, and valid for arbitrary spectral width. We show that in the limit of narrowband waves, the transport equation reduces to the linear Alber equation, which is thus a special case. Moreover, for homogeneous waves with an arbitrary spectrum, our result is consistent with the radiative transfer equation for the transport of the variance (or action) density spectrum. Comparison with analytic solutions for wave packet interference, and with observations of random surface wave propagation over a two-dimensional bottom feature, confirm that the quasi-coherent (QC) approximation accurately represents both the generation and transport of cross correlations in the wave field and resolves the finescale interference patterns associated with crossing waves. The effects of diffraction on statistics of waves around and behind obstacles and barriers can be accurately modeled by including appropriate boundary conditions on the QC approximation. These results show that the application of QC theory to ocean waves can resolve some of the restrictions of quasihomogeneous theory (the radiative transfer equation) in areas characterized by two-dimensional medium variations and caustics. This is likely to be of particular importance for wave-driven circulation and transport processes in coastal areas and inlets.

Acknowledgments. This research is supported by the U.S. Office of Naval Research (Coastal Geosciences Program and Physical Oceanography Program) and by the National Oceanographic Partnership Program. The authors thank Leo Holthuijsen whose continuing support made it possible for PBS to pursue this research.

\section{APPENDIX A}

\section{Operator Definition}

We assume that the wave field consists of a large number of progressive wave packets, and each packet $j$ has a location $\mathbf{x}_{j}(t)$, slowly varying wavenumber $\mathbf{k}_{j}(t)$, and angular frequency $\omega_{j}(t)$, which are related by a dispersion relation, $\omega_{j}=\sigma\left(\mathbf{k}_{j}, \mathbf{x}_{j}\right)$. To obtain an evolution equation for the transformed free-surface variable $\hat{\zeta}(\mathbf{k}, t)$, we associate the (dependent) wave variables $\mathbf{x}_{j}, \mathbf{k}_{j}, \omega_{j}$ with operators on $\hat{\zeta}(\mathbf{k})$, that is, $\mathbf{k}_{j}(t) \rightarrow \mathbf{k}, \mathbf{x}_{j}(t) \rightarrow i \nabla_{\mathbf{k}}$ and $\omega(t) \rightarrow i \partial_{t}$. So that the dispersion relation defines a linear operator $\Omega$ on $\hat{\zeta}$, written as

$$
\partial_{t} \hat{\zeta}(\mathbf{k}, t)=-\mathrm{i} \Omega\left(\mathbf{k}, i \nabla_{\mathbf{k}}\right) \hat{\zeta}(\mathbf{k}, t) .
$$

Although Eq. (A1) can be justified by the analogy between the ray equations of geometric optics and the canonical equations of Hamilton (e.g., Salmon 1998), the definition of the operator $\Omega$ requires particular consideration owing to noncommutability of the operators (e.g., Torre 2005). Here we follow the Weyl correspondence rule (e.g., Agarwal and Wolf 1970) to uniquely define the operator $\Omega$ so that the resulting linear operator is Hermitian with real eigenvalues (i.e., the angular frequencies), and orthogonal eigenfunctions. Thereto we expand $\sigma$ in terms of its Fourier integral,

$$
\sigma(\mathbf{k}, \mathbf{x})=\iint \hat{\sigma}(\mathbf{p}, \mathbf{q}) \exp (i \mathbf{p} \cdot \mathbf{k}+i \mathbf{q} \cdot \mathbf{x}) d \mathbf{q} d \mathbf{p}
$$

where $\hat{\sigma}(\mathbf{p}, \mathbf{q})$ denotes the Fourier transform of $\sigma(\mathbf{k}, \mathbf{x})$ with respect to $(\mathbf{p}, \mathbf{q})$. After Taylor expanding the exponential function, applying the operator correspondence, and associating any products with the sum of all its possible permutations [e.g., $\mathbf{k}_{j} \mathbf{x}_{j} \rightarrow(i / 2) \mathbf{k} \nabla_{\mathbf{k}}(\ldots)+(i / 2) \nabla_{\mathbf{k}}(\mathbf{k} \ldots)$ ], we have

$$
\Omega\left(\mathbf{k}, i \nabla_{\mathbf{k}}\right)=\iint \hat{\sigma}(\mathbf{p}, \mathbf{q}) \exp \left(i \mathbf{p} \cdot \mathbf{k}-\mathbf{q} \cdot \nabla_{\mathbf{k}}\right) d \mathbf{q} d \mathbf{p} .
$$

Here the exponential operator is defined in terms of its Taylor series expansion 


$$
\exp \left(i \mathbf{p} \cdot \mathbf{k}-\mathbf{q} \cdot \nabla_{\mathbf{k}}\right)=\sum_{|n|=0}^{\infty} \frac{1}{n !}\left(i \mathbf{p} \cdot \mathbf{k}-\mathbf{q} \cdot \nabla_{\mathbf{k}}\right)^{n},
$$

and $n$ is a multi-index. With these definitions in place, Eq. (A1) describes periodic and undamped wave motion over topography and is consistent with WKB theory (See appendix B). Moreover, for an ocean of constant depth Eq. (A1) is exact because we have $\hat{\sigma}(\mathbf{p}, \mathbf{q})=\sigma(\mathbf{k}) \delta(\mathbf{p})$, so that $\Omega\left(\mathbf{k}, i \nabla_{\mathbf{k}}\right)$ reduces to a Fourier multiplier $\sigma(\mathbf{k})$.

To demonstrate that Eq. (12) and subsequently Eq. (14) follow from the definition Eq. (A3), we introduce the sum and difference coordinates $\mathbf{k}$ and $\mathbf{u}$ in Eq. (A3) to obtain

$$
\begin{aligned}
\Omega\left(\mathbf{k} \pm \frac{1}{2} \mathbf{u}, i \nabla_{\mathbf{u}} \pm \frac{i}{2} \nabla_{\mathbf{k}}\right) \hat{\mathcal{E}}(\mathbf{k}, \mathbf{u}) & =\iint \exp \left(-\mathbf{q} \cdot \nabla_{\mathbf{u}}\right) \hat{\sigma}(\mathbf{p}, \mathbf{q}) \exp \left[i \mathbf{p} \cdot\left(\mathbf{k} \pm \frac{\mathbf{u}}{2}\right)\right] \exp \left(\mp \mathbf{q} \cdot \frac{1}{2} \nabla_{\mathbf{k}}\right) d \mathbf{q} d \mathbf{p} \hat{\mathcal{E}}(\mathbf{k}, \mathbf{u}) \\
& =\int \exp \left(-\mathbf{q} \cdot \nabla_{\mathbf{u}}\right) \hat{\sigma}\left(\mathbf{k} \pm \frac{\mathbf{u}}{2}, \mathbf{q}\right) \exp \left(\mp \mathbf{q} \cdot \frac{1}{2} \nabla_{\mathbf{k}}\right) d \mathbf{q} \hat{\mathcal{E}}(\mathbf{k}, \mathbf{u}) .
\end{aligned}
$$

We replace $\hat{\sigma}[\mathbf{k} \pm(\mathbf{u} / 2), \mathbf{q}]$ by its Taylor series expansion in $\mathbf{u}$ around $\mathbf{k}$,

$$
\hat{\Omega}^{(\infty)}\left(\mathbf{k} \pm \frac{\mathbf{u}}{2}, \mathbf{q}\right)=\sum_{|n|=0}^{\infty} \frac{1}{n !} \frac{\partial^{n} \hat{\sigma}}{\partial \mathbf{k}^{n}} \frac{( \pm \mathbf{u})^{n}}{2^{|n|}}
$$

so that, upon Fourier transforming with respect to $\mathbf{u},(\mathrm{A} 5)$ can be written as

$$
\Omega^{ \pm} \mathcal{E}(\mathbf{k}, \mathbf{x})=\int \exp (i \mathbf{q} \cdot \mathbf{x}) \hat{\Omega}^{(\infty)}\left(\mathbf{k} \mp \frac{i}{2} \nabla_{\mathbf{x}}, \mathbf{q}\right) \exp \left(\mp \mathbf{q} \cdot \frac{1}{2} \nabla_{\mathbf{k}}\right) d \mathbf{q} \mathcal{E}(\mathbf{k}, \mathbf{x}),
$$

where we used $\Omega^{ \pm}=\Omega\left[\mathbf{k} \mp(i / 2) \nabla_{\mathbf{x}}, \mathbf{x} \pm(i / 2) \nabla_{\mathbf{k}}\right]$. If $\hat{\sigma}[\mathbf{k} \pm$ $(\mathbf{u} / 2), \mathbf{q}]$ is analytic, the Taylor series approximation Eq. (A6) converges and Eq. (A7) is exact. If the series only converges on some subdomain of $\mathbf{u}$, Eq. (A7) formally only applies to contributions to $\mathcal{E}$ that originate from contributions in $\hat{\mathcal{E}}$ on that domain; the implications of this in the context of ocean waves are considered in section $4 b$.

After integration with respect to q, Eq. (A7) can be written in the form of Eq. (12), written here as

$\Omega^{ \pm} \mathcal{E}(\mathbf{k}, \mathbf{x})=\left.\exp \left( \pm \frac{i}{2} \nabla_{\tilde{\mathbf{x}}} \cdot \nabla_{\mathbf{k}}\right) \Omega^{(\infty)}\left(\tilde{\mathbf{k}} \mp \frac{i}{2} \nabla_{\mathbf{x}}, \tilde{\mathbf{x}}\right)\right|_{\tilde{\mathbf{k}}=\mathbf{k}, \tilde{\mathbf{x}}=\mathbf{x}} \mathcal{E}(\mathbf{k}, \mathbf{x})=\left.\left[\exp \left( \pm \frac{i}{2} \nabla_{\tilde{\mathbf{x}}} \cdot \nabla_{\mathbf{k}} \mp \frac{i}{2} \nabla_{\tilde{\mathbf{k}}} \cdot \nabla_{\mathbf{x}}\right) \sigma(\tilde{\mathbf{k}}, \tilde{\mathbf{x}})\right]\right|_{\tilde{\mathbf{k}}=\mathbf{k}, \tilde{\mathbf{x}}=\mathbf{x}} \mathcal{E}(\mathbf{k}, \mathbf{x})$.

Alternatively, if we observe that $\exp \left[-(1 / 2) \mathbf{q} \cdot \nabla_{\mathbf{k}}\right]$ operating on $\mathcal{E}$ is equivalent to a Taylor series of $\mathcal{E}[\mathbf{k}-(1 / 2) \mathbf{q}, \mathbf{x}]$, we can write Eq. (A7) as

$$
\Omega^{ \pm} \mathcal{E}(\mathbf{k}, \mathbf{x})=\int d \mathbf{q}\left[\exp (i \mathbf{q} \cdot \mathbf{x}) \exp \left(\mp \frac{i}{2} \nabla_{\tilde{\mathbf{k}}} \cdot \nabla_{\mathbf{x}}\right) \hat{\sigma}(\tilde{\mathbf{k}}, \mathbf{q})\right]_{\tilde{\mathbf{k}}=\mathbf{k}} \mathcal{E}\left(\mathbf{k}-\frac{1}{2} \mathbf{q}, \mathbf{x}\right),
$$

which is the operator in Eq. (14).

\section{APPENDIX B}

\section{Relation to Geometric Optics}

To show that the wave Eq. (3) [or Eq. (A1)], combined with the definition Eq. (A3), is consistent with WKB theory [to $O(\varepsilon)$ ], and describes progressive ocean waves in a slowly varying medium, we rewrite Eq. (A1) in the spatial domain, as

$$
\partial_{t} \zeta(\mathbf{x}, t)=-i \Omega\left(-i \nabla_{\mathbf{x}}, \mathbf{x}\right) \zeta(\mathbf{x}, t)
$$

We assume that the wave field is characterized by a carrier wavenumber $\mathbf{k}_{0}$, and frequency $\sigma_{0}\left(\mathbf{k}_{0}, \mathbf{x}_{0}\right)$, and write the operator $\Omega$ as 


$$
\begin{aligned}
\Omega\left(-i \nabla_{\mathbf{x}}, \mathbf{x}\right) & =\iint \hat{\sigma}(\mathbf{p}, \mathbf{q}) \exp \left(-\mathbf{p} \cdot \nabla_{\mathbf{x}}+i \mathbf{q} \cdot \mathbf{x}\right) d \mathbf{q} d \mathbf{p}=\iint \hat{\sigma}(\mathbf{p}, \mathbf{q}) \exp \left(-\frac{i}{2} \mathbf{p} \cdot \mathbf{q}\right) \exp (i \mathbf{q} \cdot \mathbf{x}) \exp \left(-i \mathbf{p} \cdot \nabla_{\mathbf{x}}\right) d \mathbf{q} d \mathbf{p} \\
& =\left.\exp \left(-\frac{i}{2} \nabla_{\tilde{\mathbf{k}}} \cdot \nabla_{\tilde{\mathbf{x}}}\right) \exp \left[-i \nabla_{\tilde{\mathbf{k}}} \cdot\left(\nabla_{\mathbf{x}}-\mathbf{k}_{0}\right)\right] \sigma(\tilde{\mathbf{k}}, \tilde{\mathbf{x}})\right|_{\tilde{\mathbf{k}}=\mathbf{k}_{0}, \tilde{\mathbf{x}}=\mathbf{x}},
\end{aligned}
$$

where the extra factor $\exp [-(i / 2) \mathbf{p} \cdot \mathbf{q}]$ in the second line appears because the products in the arguments of the exponentials do not commute, that is, $\nabla_{\mathbf{x}}(\mathbf{x} \ldots) \neq$ $\mathbf{x} \cdot \nabla_{\mathbf{x}}(\ldots)$ (see, e.g., Mesiah 1961, p. 442). We introduce the slow coordinates $T=\boldsymbol{\varepsilon} t, \mathbf{X}=\boldsymbol{\varepsilon} \mathbf{x}$, substitute the ansatz $\zeta(\mathbf{X}, T)=A(\mathbf{X}, t) \exp [i S(\mathbf{X}, T)]$ in Eq. (B1), and assume that the amplitude and phase are real and can be expanded as $A=A_{0}+\varepsilon A_{1}+\cdots$ and $S=\varepsilon^{-1} S_{0}+$ $S_{1}+\cdots$, respectively. On expanding the exponential operators in a Taylor series and retaining terms up to $O(\varepsilon)$, while dropping the scaling of the coordinates, we obtain the eikonal equation for the phase function

$$
\begin{aligned}
\partial_{\mathrm{t}} S_{0} & =-\left.\sum_{|n|=0}^{\infty} \frac{1}{n !}\left[\left(\nabla x S_{0}-k_{0}\right) \cdot \nabla_{k}\right]^{n} \sigma(\mathbf{k}, \mathbf{x})\right|_{\mathbf{k}_{0}, \mathbf{x}} \\
& =-\sigma\left(\nabla_{x} S_{0}, \mathbf{x}\right) .
\end{aligned}
$$

Similarly, the amplitude evolution equation becomes

$$
\begin{aligned}
\partial_{t} A_{0} & =-\left.\sum_{|n|=0}^{\infty} \frac{1}{n !}\left[\left(\nabla_{\boldsymbol{x}} S_{0}-\mathbf{k}_{0}\right) \cdot \nabla_{\mathbf{k}}\right]^{n}\left(\nabla_{\mathbf{x}} A_{0}+\frac{1}{2} A_{0} \nabla_{\mathbf{x}}^{2} S_{0} \nabla_{\mathbf{k}}^{2}+\frac{1}{2} A_{0} \nabla_{\mathbf{x}} \cdot \nabla_{\mathbf{k}}\right) \sigma(\mathbf{k}, \mathbf{x})\right|_{\mathbf{k}_{0}, \mathbf{x}} \\
& =-\left(\nabla_{\mathbf{k}} \sigma \cdot \nabla_{\mathbf{x}}+\frac{1}{2} \nabla_{\mathbf{k}} \cdot \nabla_{\mathbf{x}} \sigma\right) A_{0},
\end{aligned}
$$

where the derivatives of $\sigma$ are evaluated at $\left(\nabla_{\mathbf{x}} S_{0}, \mathbf{x}\right)$. If we multiply Eq. (B4) by $\rho g A_{0}$, with $\rho$ the density, and define the wave energy as $E=(1 / 2) \rho g A_{0}^{2}$, we find

$$
\partial_{t} E+\nabla_{\mathbf{x}} \cdot\left(\nabla_{\mathbf{k}} \sigma E\right)=0 .
$$

Equations (B3) and (B5) are the usual geometric optics approximations for waves in a slowly varying medium (e.g., Dingemans 1997; Mei et al. 2005), which shows that the wave Eq. (3) is consistent with WKB theory (to the order considered).

\section{APPENDIX C}

\section{Boundary Condition for Wide-Angle Diffraction}

To derive a boundary condition for the diffraction example, we use the expression derived by Janssen et al. (2008), based on a forward-scattering assumption and a Kirchhoff approximation (e.g., Born and Wolf 1999, p. 422). To transform the mutual spectrum used by Janssen et al. (2008) to $\hat{\mathcal{E}}\left(\mathbf{k}, u_{y}\right)$ used in Eq. (23), we express the frequency as a function of both $\mathbf{k}$ and $\mathbf{u}$, that is, $\omega=\omega(\mathbf{k}, \mathbf{u})$. In a homogeneous medium (this case), this can be done by noting that each contribution to the $\mathrm{CM}$ spectrum $\mathcal{E}$ involves two coherent waves $\zeta_{ \pm}=\hat{\zeta}\left(\mathbf{k}^{ \pm}\right) \exp \left(-i \omega^{ \pm} t\right)$, with wavenumbers $\mathbf{k}^{ \pm}=$ $\mathbf{k} \pm \mathbf{u} / 2$ and frequencies $\omega^{ \pm}=\sigma\left(\mathbf{k}^{ \pm}\right)$. Associated with these components we then have a beat frequency $\omega^{+}-$ $\omega^{-}$, related to the slow scale changes in time of the variance, and a mean frequency $\omega^{+} / 2+\omega^{-} / 2$, associated with the fast-scale oscillations; the former corresponds to $\omega_{\Delta}(\mathbf{k}, \mathbf{u})$ whereas the latter serves as our definition of $\omega(\mathbf{k}, \mathbf{u})$. To obtain a consistent QC approximation, we replace (as before) $\omega(\mathbf{k}, \mathbf{u})$ with its Taylor approximation, so that

$$
\omega^{(N)}(\mathbf{k}, \mathbf{u})=\sum_{|n|=0,2, \ldots}^{|n| \leq N} \frac{\mathbf{u}^{n}}{n ! 2^{|n|+1}} \frac{\partial^{n} \sigma}{\partial \mathbf{k}^{n}}
$$

Using a third-order approximation, the $\mathrm{CM}$ spectrum becomes $\hat{\mathcal{E}}\left(\mathbf{k}, u_{y}\right)=\mathcal{S}\left|d \omega^{(3)} / d k_{x}\right|$, for $k_{x}>0$ (and zero elsewhere), where

$$
\mathcal{S}\left[\omega^{(3)}, k_{x}, u_{y}\right]=\frac{1}{\pi^{2}} S_{0}\left[\omega^{(3)}\right] \exp \left(-\mathrm{i} u_{y} G_{\Delta}\right) \frac{|\mathbf{k}|^{2}+u_{y}^{2}\left(1+k_{y}^{2} k_{x}^{-2}\right)}{k_{x}^{2}-\frac{1}{4} k_{x}^{-2} k_{y}^{2} u_{y}^{2}} \frac{\sin \left[\left(-k_{y}-u_{y} / 2\right) G_{m}\right]}{\left(k_{y}+u_{y} / 2\right)} \frac{\sin \left[\left(-k_{y}+u_{y} / 2\right) G_{m}\right]}{\left(k_{y}-u_{y} / 2\right)},
$$


with $S_{0}(\omega)$ the incident, unidirectional frequency spectrum, and $G_{\Delta}=\left(G_{2}-G_{1}\right) / 2, G_{m}=\left(G_{1}+G_{2}\right) / 2$.

To approximate Eq. (23) numerically, we consider the solution on an equidistant Cartesian grid with horizontal resolution $\Delta x=\Delta y=(1 / 10) L_{p}$ (where $L_{p}$ is the peak wavelength), and define the $\mathbf{k}$-mesh as $\mathbf{k}_{i, j}=$ $(i, j) \Delta k$, with $i=1 \ldots 100, j=-200 \ldots 200$ and $\Delta k=$ $0.011 k_{p}$ (where $\left.k_{p}=2 \pi / L_{p}\right)$. For each wavenumber $\mathbf{k}_{i, j}$ the Fourier integral in Eq. (23) is approximated using a fast Fourier transform on an equidistant discrete array $[-N \ldots N] \Delta u$ for $u_{y}$ with mesh size $\Delta u=2 \pi L_{y}^{-1}$ and $L_{y}=(2 N+1) \Delta x$. Because the accuracy of the approximate transformation deteriorates for $u / k>1.5$ the contributions for these high wavenumbers are set to zero.

\section{REFERENCES}

Agarwal, G. S., and E. Wolf, 1970: Calculus for functions of noncommuting operators and general phase-space methods in quantum mechanics. I. Mapping theorems and ordering of functions of noncommuting operators. Phys. Rev., 2D, 21612186.

Aiki, H., and R. Greatbatch, 2011: Thickness-weighted mean theory for the effect of surface gravity waves on mean flows in the upper ocean. J. Phys. Oceanogr., 42, 725-747.

Alber, I. E., 1978: The effects of randomness on the stability of twodimensional surface wavetrains. Proc. Roy. Soc. London, A363, 525-546.

Bastiaans, M. J., 1979: Transport equations for the Wigner distribution function. Int. J. Opt., 26, 1265-1272.

_ 1997: Application of the Wigner distribution function in optics. The Wigner Distribution-Theory and Applications in Signal Processing, W. Mecklenbrauker and F. Hlawatsch, Eds., Elsevier Science, 375-426.

Berkhoff, J., N. Booy, and A. Radder, 1982: Verification of numerical wave propagation models for simple harmonic linear water waves. Coastal Eng., 6, 255-279.

Booij, N., R. C. Ris, and L. H. Holthuijsen, 1999: A thirdgeneration wave model for coastal regions. 1. Model description and validation. J. Geophys. Res., 104 (C4), 76497666.

Born, M., and E. Wolf, 1999: Principles of Optics. 7th ed. Cambridge University Press, $836 \mathrm{pp}$.

Bremmer, H., 1972: General remarks concerning theories dealing with scattering and diffraction in random media. Radio Sci., 8 , 511-534.

Cohen, L., 1989: Time-frequency distributions-A review. Proc. IEEE, 77, 941-981.

_ _ 2010: Phase-space differential equations for modes. PseudoDifferential Operators: Complex Analysis and Partial Differential Equations, B. Schulze and M. W. Wong, Eds., Birkhäuser Basel, 235-250.

Craik, A. D. D., and S. Leibovich, 1976: A rational model for Langmuir circulations. J. Fluid Mech., 73, 401-426.

Dingemans, M., 1997: Water Wave Propagation over Uneven Bottoms. Advanced Series on Ocean Engineering, Vol. 23, World Scientific, $471 \mathrm{pp}$.
Dysthe, K. B., 1979: Note on a modification to the nonlinear schrodinger equation for application to deep water waves. Proc. Roy. Soc. London, A369, 105-114.

Hlawatsch, F., and P. Flandrin, 1997: The interference structure of the wigner distribution and related time-frequency signal representations. The Wigner Distribution-Theory and Applications in Signal Processing, W. Mecklenbrauker and F. Hlawatsch, Eds., Elsevier, 59-133.

Hoefel, F., and S. Elgar, 2003: Wave-induced sediment transport and sandbar migration. Science, 299, 1885-1887.

Janssen, A., and T. Claasen, 1985: On positivity of time-frequency distributions. IEEE Trans. Acoust. Speech Signal Process., 33, $1029-1032$

Janssen, P., 2009: The Interaction of Ocean Waves and Wind. Cambridge University Press, $300 \mathrm{pp}$.

Janssen, T. T., and T. H. C. Herbers, 2009: Nonlinear wave statistics in a focal zone. J. Phys. Oceanogr., 39, 1948-1964.

- _ - and J. A. Battjes, 2008: Evolution of ocean wave statistics in shallow water: Refraction and diffraction over seafloor topography. J. Geophys. Res., 113, C03024, doi:10.1029/ 2007JC004410.

Komen, G., L. Cavaleri, M. Donelan, K. Hasselmann, S. Hasselmann, and P. Janssen, 1994: Dynamics and Modelling of Ocean Waves. Cambridge University Press, 532 pp.

Kuik, A., G. van Vledder, and L. Holthuijsen, 1988: A method for the routine analysis of pitch-and-roll buoy wave data. J. Phys. Oceanogr., 18, 1020-1034.

Magne, R., K. A. Belibassakis, T. H. C. Herbers, F. Ardhuin, W. C. O'Reilly, and V. Rey, 2007: Evolution of surface gravity waves over a submarine canyon. J. Geophys. Res., 112, C01002, doi:10.1029/2005JC003035.

Mandel, L., and E. Wolf, 1995: Optical Coherence and Quantum Optics. Cambridge University Press, $1166 \mathrm{pp}$

McWilliams, J. C., and J. M. Restrepo, 1999: The wavedriven ocean circulation. J. Phys. Oceanogr., 29, 25232540.

Mei, C., M. Stiassnie, and D. Yue, 2005: Theory and Applications of Ocean Surface Waves. Advanced Series on Ocean Engineering, Vol. 21, World Scientific, 503 pp.

Mesiah, A., 1961: Quantum Mechanics: Vol. I. North-Holland Publishing Company, 1136 pp.

O'Reilly, W. C., and R. T. Guza, 1991: Comparison of spectral refraction and refraction-diffraction wave models. J. Waterw. Port Coastal Ocean Eng., 117, 199-215.

Penney, W. G., and A. T. Price, 1952: The diffraction theory of sea waves and the shelter afforded by breakwaters. Part I. Proc. Roy. Soc. London, A244, 236-253.

Salmon, R., 1998: Lectures on Geophysical Fluid Dynamics. Oxford University Press, $400 \mathrm{pp}$.

Smith, J. A., 2006: Observed variability of ocean wave Stokes drift, and the Eulerian response to passing groups. J. Phys. Oceanogr., 36, 1381-1402.

Stamnes, J., 1986: Waves in Focal Regions: Propagation, Diffraction, and Focusing of Light, Sound, and Water Waves. A. Hilger, $618 \mathrm{pp}$.

Strichartz, R., 1993: A Guide to Distribution Theory and Fourier Transforms. CRC Press, 224 pp.

Svendsen, I. A., 2006: Introduction to Nearshore Hydrodynamics. Advanced Series on Ocean Engineering, Vol. 24, World Scientific, $744 \mathrm{pp}$.

The WAMDI Group, 1988: The WAM model-A third generation ocean wave prediction model. J. Phys. Oceanogr., 18, 17751810. 
Tolman, H. L., 1991: A third-generation model for wind waves on slowly varying, unsteady, and inhomogeneous depths and currents. J. Phys. Oceanogr., 21, 782-797.

Torre, A., 2005: Linear Ray and Wave Optics in Phase Space: Bridging Ray and Wave Optics via the Wigner Phase-Space. Elsevier, 540 pp.

Ville, J., 1948: Thorie et applications de la notion de signal analytique. Cables Transm., 2A, 61-74.

Vincent, C. L., and M. J. Briggs, 1989: Refraction-diffraction of irregular waves over a mound. J. Waterw. Port Coastal Ocean Eng., 115, 269-284.
Walther, A., 1968: Radiometry and coherence. J. Opt. Soc. Amer., 58, 1256-1259.

Wigner, E., 1932: On the quantum correction for thermodynamic equilibrium. Phys. Rev., 40, 749-759.

Wise Group, 2007: Wave modeling-The state of the art. Prog. Oceanogr., 75, 603-674.

Zijlema, M., G. Stelling, and P. Smit, 2011: SWASH: An operational public domain code for simulating wave fields and rapidly varied flows in coastal waters. Coastal Eng., 58, 9921012. 